\title{
Enumerating all Hamilton Cycles and Bounding the Number of Hamilton Cycles in 3-Regular Graphs *
}

\author{
Heidi Gebauer \\ Institute of Theoretical Computer Science \\ ETH Zurich, CH-8092 Zurich, Switzerland \\ gebauerh@inf .ethz.ch
}

Submitted: Sep 22, 2009; Accepted: Jun 10, 2011; Published: Jun 21, 2011

Mathematics Subject Classifications: 05C35, 05C45, 05C85

\begin{abstract}
We describe an algorithm which enumerates all Hamilton cycles of a given 3regular $n$-vertex graph in time $O\left(1.276^{n}\right)$, improving on Eppstein's previous bound. The resulting new upper bound of $O\left(1.276^{n}\right)$ for the maximum number of Hamilton cycles in 3-regular $n$-vertex graphs gets close to the best known lower bound of $\Omega\left(1.259^{n}\right)$. Our method differs from Eppstein's in that he considers in each step a new graph and modifies it, while we fix (at the very beginning) one Hamilton cycle $C$ and then proceed around $C$, successively producing partial Hamilton cycles.
\end{abstract}

\section{Introduction}

The famous traveling salesman problem (TSP) is one of the most fundamental NPcomplete graph problems [4]. For decades the best known algorithm for TSP was the dynamic programming algorithm by Held and Karp [6], which runs in time $O\left(2^{n}\right)$ with $n$ denoting the number of vertices of the given graph. This was also the strongest known upper bound for the subproblem of deciding whether a given graph contains a Hamilton cycle. In a recent breakthrough, Björklund [2] gave a Monte Carlo algorithm for detecting whether a given graph contains a Hamilton cycle or not which runs in time $1.657^{n}$ poly $(n)$, with false positives and false negatives occurring with probability exponentially small in $n$. (We let "poly $(n)$ " denote a polynomial factor in $n$.) For bipartite graphs this algorithm even runs in time $2^{\frac{n}{2}}$ poly $(n)$.

Despite this major development it is still open whether the traveling salesman problem in its general form can be solved in time $O\left(1.999^{n}\right)$ [9]. Therefore it is of interest to consider some restricted problem classes, which - while still NP-complete - might be treated faster.

\footnotetext{
${ }^{*}$ An extended abstract appeared in Proc. 5th Workshop on Analytic Algorithmics and Combinatorics (ANALCO) (2008)
} 
3-regular graphs Eppstein established an algorithm which solves the traveling salesman problem in $O\left(2^{\frac{n}{3}}\right)\left(2^{\frac{1}{3}} \approx 1.260\right)$. He additionally showed that this algorithm can be modified to enumerate all Hamilton cycles in time $O\left(2^{\frac{3 n}{8}}\right) \leq 1.297^{n}$. This value is also the best known upper bound for the number of Hamilton cycles in 3-regular graphs. The corresponding algorithm basically solves the more general problem of listing all Hamilton cycles which contain a given set of forced edges. In each step it recursively deletes some edges and marks others as "forced" and then continues with the resulting, new graph.

Iwama and Nakashima [7] reduced Eppstein's time upper bound of $O\left(2^{\frac{n}{3}}\right)$ to $O\left(1.251^{n}\right)$.

We note that all the results mentioned above were originally stated for the class of maximum-degree-3 graphs.

4-regular graphs Eppstein also gave a randomized reduction from maximum-degree-4 graphs to maximum-degree-3 graphs, which allows to solve the traveling salesman problem for a given 4-regular graph $G$ in time $O\left(\left(\frac{3}{2}\right)^{n} \cdot t_{3}(n)\right)$ with $t_{3}(n)$ denoting the time needed to solve the traveling salesman problem for graphs of maximum degree 3 . By the result of Iwama and Nakashima this is bounded by $O\left(1.876^{n}\right)$. In [5] we improve this upper bound to $\sqrt{3}^{n} \operatorname{poly}(n)(\sqrt{3} \approx 1.732)$ and show that all Hamilton cycles can be listed in time $O\left(1.783^{n}\right)$.

Graphs of maximum degree $k$ By modifying the classical Bellman-Held-Karp algorithm Björklund, Husfeldt, Kaski and Koivisto [1] showed that the traveling salesman problem for a graph with maximum degree $k$ can be solved in $O\left(\left(2^{(k+1)}-2 k-2\right)^{n /(k+1)}\right)$. For 3-regular and 4-regular graphs the resulting bounds are weaker than the known bounds, however, for $k \geq 5$ this improves the previously best upper bound of $O\left(2^{n}\right)$.

Our contribution We improve Eppstein's time upper bound of $O\left(2^{\frac{3 n}{8}}\right)\left(2^{\frac{3}{8}} \approx 1.297\right)$ for listing all Hamilton cycles to $O\left(1.276^{n}\right)$. The resulting new upper bound of $O\left(1.276^{n}\right)$ for the maximum number of Hamilton cycles in 3-regular graphs gets close to the corresponding lower bound of $2^{\frac{n}{3}}\left(2^{\frac{1}{3}} \approx 1.260\right)$ shown by Eppstein. It is important to note that our method is not a refinement of Eppstein's procedure but a new approach. Whereas Eppstein in each step considers a new graph and recursively modifies it we let the original graph stay as it is (throughout the whole algorithm) - at the beginning we fix one Hamilton cycle $C$ and then proceed around $C$, successively producing partial Hamilton cycles.

We finally remark that every algorithm $A$ which enumerates all Hamilton cycles of a 3-regular graph on $n$ vertices in time $T(n)$ can also be used to enumerate all Hamilton cycles of a graph with degree at most 3 in time $T(n) \cdot \operatorname{poly}(n)$. Indeed, if the given graph $G$ has a vertex of degree at most one then $G$ does not have a Hamilton cycle. Otherwise, let $G^{\prime}$ be the graph obtained by replacing every maximal path $P=v_{1}, \ldots, v_{k}$ of degreetwo vertices with an edge $e_{P}$ connecting the (degree-3) neighbor of $v_{1}$ with the (degree-3) neighbor of $v_{k}$. Note that the Hamilton cycles of $G$ correspond to the Hamilton cycles of $G^{\prime}$ containing every edge of the form $e_{P}$. By enumerating all Hamilton cycles of $G^{\prime}$ and ignoring those which do not contain every edge of the form $e_{P}$ we obtain a list of all 

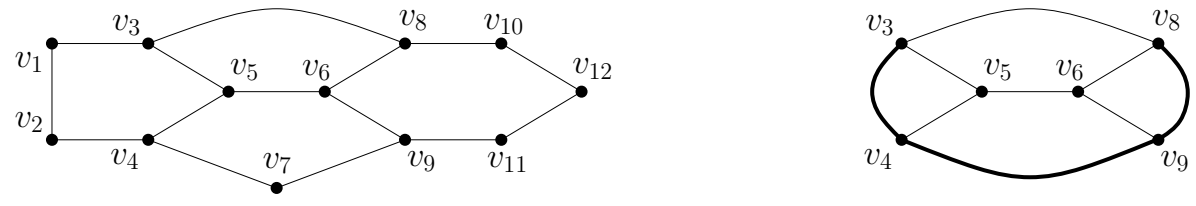

Figure 1: An example for the transformation of a graph $G$ (on the left) into a 3-regular graph $G^{\prime}$ (on the right). The edges of the form $e_{P}$ are drawn thick.

Hamilton cycles of $G^{1}$. Hence our algorithm can also enumerate all Hamilton cycles of a given graph of maximum degree 3 in time at most $O\left(1.276^{n}\right)$.

Lower bounds Eppstein exhibited an infinite family of 3-regular graphs with $2^{\frac{n}{3}}\left(2^{\frac{1}{3}} \approx\right.$ 1.260) Hamilton cycles per graph, implying a lower bound of $2^{\frac{n}{3}}$ for the maximum number of Hamilton cycles in 3-regular graphs. Eppstein conjectures that this is tight, i.e., that every 3-regular graph on $n$ vertices has at most $2^{\frac{n}{3}}$ Hamilton cycles. While this conjecture is still open we can refute it for the class of graphs of average degree 3: In [5] we construct for every $n$ divisible by 8 a 4 -regular graph $G_{n}$ such that the number of Hamilton cycles in $G_{n}, \mathrm{hc}\left(G_{n}\right)$, is $48^{\frac{n}{8}} \geq 1.622^{n}$. For every $n$ divisible by 16 we fix a vertex $v$ of $G_{\frac{n}{2}}$. By a straightforward average argument some edge $e$ incident to $v$ occurs in at least a quarter of all Hamilton cycles of $G_{\frac{n}{2}}$. We add $\frac{n}{2}$ vertices to $e$ and let $H_{n}$ denote the resulting graph. $H_{n}$ is an $n$-vertex graph of average degree 3 with $\mathrm{hc}\left(H_{n}\right) \geq \frac{1}{4} \cdot \mathrm{hc}\left(G_{\frac{n}{2}}\right) \geq \frac{1}{4} \cdot 48^{\frac{n / 2}{8}} \geq 1.273^{n}$ for $n$ large enough. If Eppstein's Conjecture is true this implies that the average-degree-3 graph maximizing the number of Hamilton cycles is not 3-regular. If it is not possible to prove Eppstein's Conjecture it would already be interesting to know whether one can separate the average-degree-3 case from the 3-regular case, i.e., whether one can show that the maximum number of Hamilton cycles in 3-regular graphs is strictly smaller than the maximum number of Hamilton cycles in graphs of average degree 3 .

Multigraphs A multigraph is a graph which - in contrast to ordinary graphs - is allowed to have loops and multiple edges. Sharir and Welzl [8] implicitly showed that every 3regular multigraph has at most $\sqrt{2}^{n}$ Hamilton cycles and they also gave an algorithm which lists all Hamilton cycles in time $\sqrt{2}^{n}$ poly $(n)$. This bound is tight, since the graph $G$ obtained by taking a cycle $v_{1}, \ldots, v_{n}, v_{1}$ for an even number $n$, and adding an extra edge $\left(v_{i}, v_{i+1}\right)$ for every odd $i$ with $1 \leq i \leq n-1$, has exactly $2^{\frac{n}{2}}$ Hamilton cycles (indeed,

\footnotetext{
${ }^{1}$ We note for completeness that the case where the addition of the $e_{P}$ lead to a loop or to multiple edges needs a special treatment: Suppose first that $G^{\prime}$ consists of at most two vertices. Then we can easily enumerate all Hamilton cycles by hand. So let $G^{\prime}$ be a graph on at least 3 vertices. If some $e_{P}$ forms a loop in $G^{\prime}$ or if two edges $e_{P}, e_{P^{\prime}}$ are parallel in $G^{\prime}$ then $G$ has no Hamilton cycle. Finally, if some $e_{P}$ has a parallel edge $e$ which is not of the form $e_{P^{\prime}}$ then deleting $e$ in $G^{\prime}$ does not reduce the set of Hamilton cycles we are interested in. So it suffices to list the Hamilton cycles of the graph $G^{\prime \prime}$ obtained by deleting every edge $e$ which has a parallel edge of the form $e_{P}$. (Note that since $G^{\prime \prime}$ is not necessarily 3 -regular we might need to recursively apply the steps described above.)
} 
for every even $i$ we have to include $\left(v_{i}, v_{i+1}\right)$ and for every odd $i$ we can choose between the two edges connecting $v_{i}$ and $v_{i+1}$ ). So we cannot hope to obtain a faster algorithm for listing all Hamilton cycles in multigraphs.

Notation Let $G$ be a graph. An ordering $\sigma=v_{1}, v_{2}, \ldots, v_{n}$ of the vertices of $G$ is called a Hamilton ordering if $v_{1}, v_{2}, \ldots, v_{n}, v_{1}$ is a Hamilton cycle. For a given Hamilton ordering $\sigma=v_{1}, v_{2}, \ldots, v_{n}$ of the vertices, we call an edge $e$ a diagonal if $e$ is not on the cycle $v_{1}, v_{2}, \ldots, v_{n}, v_{1}$, and we call a vertex $v_{i}$ active if it is adjacent to a vertex $v_{j}$ with $j \geq i+2$. A vertex which is not active is called passive.

Organization of this paper In Section 2 we describe our algorithm to enumerate all Hamilton cycles in a 3-regular graph. In Section 3 we give some definitions and general facts. In Section 4 we state two key lemmas, which help us to analyze the running time of our algorithm, and show that they imply the following theorem.

Theorem 1.1. The Hamilton cycles of a given 3-regular n-vertex graph $G$ can be enumerated in time

$$
1.628^{\frac{n}{2}} \cdot \operatorname{poly}(n)=\mathcal{O}\left(1.276^{n}\right) \text {. }
$$

Section 5 and 6 deal with the proofs of the two key lemmas.

\section{The Algorithm}

First we test whether $G$ is Hamiltonian and, if yes, construct one Hamilton cycle. This can be done rather quickly due to known algorithms for finding the minimum weight Hamilton cycle, established, for example, by Eppstein [3], and Iwama and Nakashima [7]. Their algorithms have running time $\mathcal{O}\left(1.260^{n}\right)$ and $\mathcal{O}\left(1.251^{n}\right)$, respectively, which, compared to the claimed bound of $\mathcal{O}\left(1.276^{n}\right)$, is negligible. So we have the following.

Observation 2.1. We can obtain a Hamilton ordering $\sigma=v_{1}, \ldots, v_{n}$ of the vertices of $G$ in time $\mathcal{O}\left(1.251^{n}\right)$.

The basic idea of our algorithm is the following. First we take the Hamilton ordering $\sigma=v_{1}, \ldots, v_{n}$ given by Observation 2.1 (possibly with slight modifications). Then we consider the following procedure for constructing another Hamilton cycle $H$ : We process the vertices $v_{1}, \ldots, v_{n-1}$ one by one and carefully decide for each vertex $v_{i}$ which of its outgoing edges are included in $H$. It will turn out that there are many vertices where we have only one option to decide on, implying that the number of outcomes of our procedure (i.e. Hamilton cycles and attempts where we get stuck) is rather small.

We now give a more formal description of the above. We fix a Hamilton ordering $v_{1}, \ldots, v_{n}$ of the vertices of $G$ and direct each edge - except for $\left(v_{n}, v_{1}\right)$ - from the vertex with the lower index to the vertex with the higher index. (Figure 2 shows an example.) Let $\left(v_{i}, v_{j}\right)$ be a diagonal with $i<j$ (recall that a diagonal is an edge which is not on the cycle $\left.v_{1}, \ldots, v_{n}, v_{1}\right)$. Then $\left(v_{i}, v_{j}\right)$ is an outgoing diagonal of $v_{i}$, and an incoming diagonal 


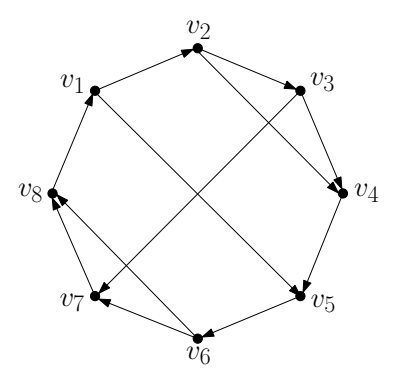

Figure 2: An example for $n=8$. Here $v_{1}, v_{2}, v_{3}, v_{6}$ are active while $v_{4}, v_{5}, v_{7}, v_{8}$ are passive.

of $v_{j}$. Note that the outdegree of every vertex is either one or two. The following is a direct consequence of our definition of active and passive vertices.

Remark. A vertex is active if it has outdegree two, otherwise it is passive.

We will see that when we process an active vertex $v_{i}$ in our procedure for constructing another Hamilton cycle then we might have more than one option to decide which of the outgoing edges of $v_{i}$ to include.

Remark 2.2. $v_{1}$ and $v_{2}$ are active (since they can not have an incoming diagonal) whereas $v_{n-1}$ and $v_{n}$ are passive (since they can not have an outgoing diagonal). Since the edges which are diagonals constitute a matching in $G$ there are $\frac{n}{2}$ diagonals. Thus there are $\frac{n}{2}$ active vertices in total.

We now describe the procedure $P_{\text {ham }}$ for constructing another Hamilton cycle. For every vertex $v_{i} \in\left\{v_{1}, \ldots, v_{n}\right\}$ we will select some of its outgoing edges, and we will maintain a set $S$ which contains all edges that have been selected so far.

Procedure $P_{\text {ham }}$ First we decide whether or not to select $\left(v_{n}, v_{1}\right)$. Then we process the vertices $v_{1}, \ldots, v_{n-1}$ one by one. We refer to the processing of $v_{i}$ by round $i$. In round $i$ we carefully select some outgoing edges of $v_{i}$ such that afterwards the following holds.

(i) Each vertex $v_{j}$ with $j \leq i$ is incident to exactly two selected edges.

(ii) The set of selected edges does not contain a cycle of length smaller than $n$.

(iii) If $v_{i+1}$ has two incoming edges then at least one of them must be selected.

We call (i) - (iii) the postconditions (for round i). Note that these conditions only filter out selections which can not be completed to a Hamilton cycle. If it is not possible to select some of the outgoing edges of $v_{i}$ such that postcondition (i) - (iii) are fulfilled then we give up and stop. We now have a closer look at round $i$. 
Processing of $v_{i}$ We distinguish two cases.

Case 1: $v_{i}$ is passive. In this case there is only one option.

Indeed, since postcondition (iii) is satisfied after round $i-1$, at least one of the incoming edges of $v_{i}$ is selected. If both incoming edges are selected then we do not select the outgoing edge of $v_{i}$ (the only way to fulfill postcondition (i)), otherwise (also due to postcondition (i)) we select the outgoing edge of $v_{i}$. (It is of course possible that our selection violates postcondition (ii) or (iii); in this case we give up and stop.)

Case 2: $v_{i}$ is active. In this case there might or might not be two options.

Let $d$ denote the outgoing diagonal of $v_{i}$. If the incoming edge of $v_{i}$ is not selected then (by postcondition (i)) we select both of its outgoing edges and check whether postcondition (ii) and (iii) are fulfilled (if this is not the case we give up and stop). Otherwise we select one edge of $\left\{\left(v_{i}, v_{i+1}\right), d\right\}$ such that postcondition (ii) and (iii) are satisfied (if this is not possible we give up and stop).

If we did not give up we continue with $v_{i+1}$ and go on. After processing $v_{n-1}$ we check whether the set $S$ of selected edges forms a Hamilton cycle. If yes, we output $S$, otherwise we do nothing.

Algorithm for enumerating all Hamilton cycles Note that every Hamilton cycle can be obtained as an outcome of $P_{\text {ham }}$ (with the appropriate selections). So the algorithm $\mathcal{A}$ which goes through all possible outcomes of $P_{\text {ham }}$ will list all Hamilton cycles. (Note that $\mathcal{A}$ can easily be implemented, e.g., using backtracking.) We now bound the running time of $\mathcal{A}$.

Definition 2.3. Let $v_{1}, \ldots, v_{n}$ be a Hamilton ordering of the vertices of $G$ and let $1 \leq$ $i \leq n-1$. Each edge set which can be obtained by performing $i$ rounds of $P_{\text {ham }}$ will be called a choice for $v_{1}, \ldots, v_{i}$. With $\mathrm{ch}\left(v_{i}\right)$ we denote the set of choices for $v_{1}, \ldots, v_{i}$.

By a slight abuse of notation we let $\operatorname{ch}\left(v_{0}\right)$ denote the set of choices for the very first decision (directly before round 1) and so $\mathrm{ch}\left(v_{0}\right)$ consists of the empty set and the set containing only the edge $\left(v_{n}, v_{1}\right)$.

Let $S$ be an edge set which is an outcome of $P_{\text {ham }}$. Then either $S$ forms a Hamilton cycle or we gave up after having selected the edges in $S$. In the former case, $S \in \operatorname{ch}\left(v_{n-1}\right)$, whereas in the latter case, $S \in \operatorname{ch}\left(v_{i}\right)$ for some $i \leq n-1$. So the number of outcomes of $P_{\text {ham }}$ is bounded by $\left|\operatorname{ch}\left(v_{1}\right)\right|+\left|\operatorname{ch}\left(v_{2}\right)\right|+\ldots+\left|\operatorname{ch}\left(v_{n-1}\right)\right|$. Since one iteration of $P_{\text {ham }}$ can be done in time polynomial in $n$ we get the following.

Observation 2.4. For every given Hamilton ordering $v_{1}, \ldots, v_{n}$ of the vertices of $G$ the algorithm $\mathcal{A}$ runs in time at most $\left(\left|\operatorname{ch}\left(v_{1}\right)\right|+\left|\operatorname{ch}\left(v_{2}\right)\right|+\ldots+\left|\operatorname{ch}\left(v_{n-1}\right)\right|\right) \cdot \operatorname{poly}(n)$.

Finding an appropriate Hamilton ordering of the vertices We now carefully choose an ordering of the vertices which allows us to prove the claimed upper bound on the running time of $\mathcal{A}$. We first identify certain patterns which are beneficial and disadvantageous, respectively, for our analysis of the running time of $\mathcal{A}$. 

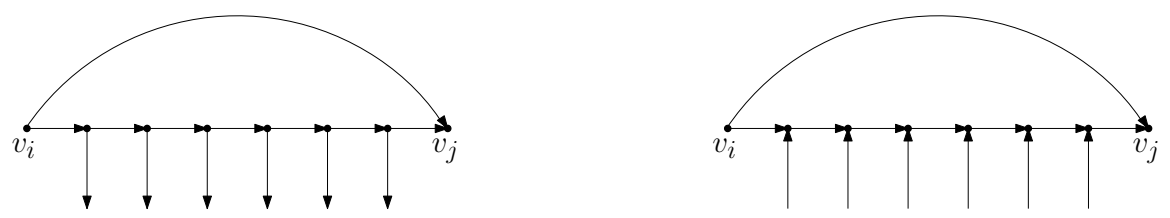

Figure 3: An outward pattern (on the left) and an inward pattern (on the right).

Definition 2.5. Let $v_{1}, \ldots, v_{n}$ be a Hamilton ordering of the vertices of $G$. We call a sequence $\left(v_{i}, v_{i+1}, \ldots, v_{j}\right)$ with $1 \leq i<i+2 \leq j \leq n$, (i) an outward pattern if there is a diagonal $\left(v_{i}, v_{j}\right)$ and $v_{i}, v_{i+1} \ldots, v_{j-1}$ are all active, (ii) an inward pattern if there is a diagonal $\left(v_{i}, v_{j}\right)$ and $v_{i+1}, v_{i+2}, \ldots, v_{j}$ are all passive.

Figure 3 depicts an outward pattern and an inward pattern. From now on we consider a fixed Hamilton ordering $v_{1}, \ldots, v_{n}$ (by Observation 2.1 such an ordering can be found quickly enough). It will turn out that inward patterns have a rather bad influence on the running time of our algorithm whereas outward patterns have a good influence. So the next observation is crucial.

Observation 2.6. We can assume that the number of outward patterns is at least the number of inward patterns.

This can easily be achieved by possibly reversing the numbering of the vertices, by which inward patterns become outward patterns and vice versa. Note that the number of outward patterns and the number of inward patterns can readily be computed in polynomial time.

We now state two simple but useful properties of outward patterns. Let $v_{i}$ be a vertex of an outward pattern $P$ and let $j$ be the smallest index in $\{i, \ldots, n\}$ such that $v_{j}$ is passive. Then $P=\left(v_{k}, \ldots, v_{j}\right)$ where $v_{k}$ is the source of the incoming diagonal of $v_{j}$. Thus $P$ is uniquely determined and we have the following.

Observation 2.7. Every vertex belongs to at most one outward pattern.

Let $v_{k}$ be a passive vertex which belongs to an outward pattern $P$. Then $v_{k-1}$ also belongs to $P$ and is active. By Remark 2.2 this directly implies the following.

Observation 2.8. $v_{n}$ does not belong to an outward pattern.

Finally, we identify those vertices which make our analysis of the running time of $\mathcal{A}$ a bit more complicated, and we relate them to inward patterns.

Definition. An active vertex $v_{i}$ with $i \geq 2$ is called unpleasant if the outgoing diagonal of the previous active vertex $v_{j}$ points to a vertex in $\left\{v_{j+2}, \ldots, v_{i-1}\right\}$. An active vertex which is not unpleasant is called pleasant. In particular, $v_{1}$ is pleasant. 


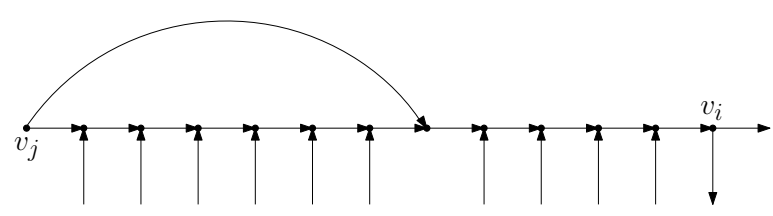

Figure 4: An unpleasant vertex $v_{i}$.

Let $v_{i}$ be an unpleasant vertex and let $v_{j}$ be its previous active vertex. Then the outgoing diagonal of $v_{j}$ points to a vertex $v_{k}$ in $\left\{v_{j+2}, \ldots, v_{i-1}\right\}$ and so $\left(v_{j}, \ldots, v_{k}\right)$ is an inward pattern. So every unpleasant vertex corresponds to an inward pattern, and therefore the number of unpleasant vertices is at most the number of inward patterns. By Observation 2.6 we get the following.

Observation 2.9. The number of unpleasant vertices is at most the number of outward patterns.

Observation 2.9 is crucial for our analysis since it allows us to compensate (to a certain extent) the bad effect of the unpleasant vertices with the good effect of the outward patterns.

A rough sketch of the analysis of the algorithm We will basically choose an appropriate constant $c>1$ and inductively show that for every $i \in\{1, \ldots, n-1\}$ we have $\left|\operatorname{ch}\left(v_{i}\right)\right| \leq 1.628^{a} \cdot c^{u} \cdot c^{-p}$ where $a, u$, and $p$, respectively, denote the number of active vertices, unpleasant vertices, and outward patterns, respectively, in $\left\{v_{1}, \ldots, v_{i}\right\}$. By Remark 2.2, Observation 2.8 and Observation 2.9 this immediately gives that $\left|\operatorname{ch}\left(v_{n-1}\right)\right| \leq$ $1.628^{\frac{n}{2}}$. A more careful analysis will allow us to show that the expression $1.628^{a} \cdot c^{u} \cdot c^{-p}$ is maximized when $i=n-1$. This implies that $\left|\operatorname{ch}\left(v_{i}\right)\right| \leq 1.628^{\frac{n}{2}}$ for every $i \in\{1, \ldots, n-1\}$, which together with Observation 2.4 implies Theorem 1.1.

\section{Definitions and General Facts}

Since we will frequently deal with choices we first state some definitions and auxiliary facts.

Observation 3.1. Let $C \in \operatorname{ch}\left(v_{k}\right)$. By postcondition (i), $v_{i}$ is incident to exactly two edges in $C$ for every $i \leq k$. By postcondition (ii), $C$ does not contain a cycle of length smaller than $n$. Finally, if $v_{k+1}$ is passive then by postcondition (iii), $C$ contains an incoming edge of $v_{k+1}$.

We will partition the sequence $v_{1}, \ldots, v_{n-1}$ into suitable subsequences and then reduce our original claim to a statement on subsequences. Therefore we extend the notion of a choice to subsequences. 
Definition 3.2. Let $C \in \operatorname{ch}\left(v_{k}\right)$ and let $D$ be any choice. $D$ is an extension of $C$ if for every outgoing edge $e$ of a vertex in $\left\{v_{n}\right\} \cup\left\{v_{1}, \ldots, v_{k}\right\}$ it holds: $e \in D$ if and only if $e \in C$.

Moreover, for every $q \geq k$ we let $\operatorname{ch}_{\mid C}\left(v_{q}\right)$ denote the set of all choices in $\operatorname{ch}\left(v_{q}\right)$ which are an extension of $C$. In particular, $\operatorname{ch}_{\mid C}\left(v_{k}\right)=\{C\}$.

So we get the following (recall that by Definition 2.3 we have $\operatorname{ch}\left(v_{0}\right)=\left\{\emptyset,\left\{\left(v_{n}, v_{1}\right)\right\}\right\}$ ).

Observation 3.3. Let $0 \leq i \leq j \leq n-1$. Every choice $D \in \operatorname{ch}\left(v_{j}\right)$ is an extension of some choice $C \in \operatorname{ch}\left(v_{i}\right)$. In particular, $\left|\operatorname{ch}\left(v_{j}\right)\right|=\sum_{C \in \operatorname{ch}\left(v_{i}\right)}\left|\operatorname{ch}_{\mid C}\left(v_{j}\right)\right|$.

Focussing on a subset of the vertices Let $i_{\max }$ denote the index $i$ which maximizes $\left|\operatorname{ch}\left(v_{i}\right)\right|$. The following is a direct consequence of Observation 2.4.

Observation 3.4. $\mathcal{A}$ runs in time at most $\left|\operatorname{ch}\left(v_{i_{\max }}\right)\right| \cdot \operatorname{poly}(n)$.

So our goal is to bound $\left|\operatorname{ch}\left(v_{i_{\max }}\right)\right|$. To this end we first give some basic properties of $\left\{v_{1}, \ldots, v_{i_{\max }}\right\}$, and then reduce Theorem 1.1 to two key lemmas. Let $a$ and $a^{\prime}$ denote the number of active vertices in $\left\{v_{1}, \ldots, v_{i_{\max }}\right\}$, and in $\left\{v_{i_{\max }+1}, \ldots, v_{n-1}\right\}$, respectively. By Remark 2.2 we get that

$$
a+a^{\prime}=\frac{n}{2} .
$$

Let $u_{\text {tot }}$ denote the number of unpleasant vertices in $\left\{v_{1}, \ldots, v_{n-1}\right\}$. Moreover, let $s, s^{\prime}$, and $s_{\text {tot }}$, respectively, denote the number of outward patterns fully contained in $\left\{v_{1}, \ldots, v_{i_{\max }}\right\}$, in $\left\{v_{i_{\max }+1}, \ldots, v_{n-1}\right\}$, and in $\left\{v_{1}, \ldots, v_{n-1}\right\}$, respectively. By Observation 2.8, $s_{\text {tot }}$ is the number of outward patterns. Observation 2.9 gives that

$$
u_{\text {tot }} \leq s_{\text {tot }} .
$$

By Observation 2.7 there is at most one outward pattern which contains both $v_{i_{\max }}$ and $v_{i_{\max }+1}$. Hence at least $s_{\text {tot }}-1$ patterns are either fully contained in $\left\{v_{1}, \ldots, v_{i_{\max }}\right\}$ or fully contained in $\left\{v_{i_{\max }+1}, \ldots, v_{n-1}\right\}$. So,

$$
s_{\text {tot }} \leq s+s^{\prime}+1 .
$$

\section{Reduction of Theorem 1.1 to Two Key Lemmas}

In this section we state two key lemmas and show that they imply Theorem 1.1. From now on by a pattern we mean an outward pattern fully contained in $v_{1}, \ldots, v_{i_{\max }}$. We partition the active vertices in $v_{1}, \ldots, v_{i_{\max }}$ into disjoint sets $W_{1}, \ldots, W_{k}$ where $k$ is the number of patterns plus the number of active vertices in $v_{1}, \ldots, v_{k}$ not belonging to a pattern. Each $W_{i}$ contains either all active vertices of a pattern or a single active vertex which does not belong to a pattern. Note that $v_{1} \in W_{1}$.

Definition 4.1. For $i=1, \ldots, k$ we let $f(i)$ denote the $v$-index of the first vertex in $W_{i}$ and we let $l(i)$ denote the $v$-index of the last vertex in $W_{i}$. We define $f(k+1):=i_{\max }+1$. 
Note that $W_{i}=\left\{v_{f(i)}, v_{f(i)+1}, \ldots, v_{l(i)}\right\}$ and $f(1)=1$.

Definition. For $r=0, \ldots, k$ we let $A_{r}$ ( $B_{r}$, respectively) denote the number of elements of $\operatorname{ch}\left(v_{f(r+1)-1}\right)$ which contain (do not contain, respectively) $\left(v_{f(r+1)-1}, v_{f(r+1)}\right)$. (By a slight abuse of notation we consider $\left(v_{0}, v_{1}\right)$ as the edge $\left(v_{n}, v_{1}\right)$.)

So for every $r, r=0, \ldots, k$ we have

$$
A_{r}+B_{r}=\left|\operatorname{ch}\left(v_{f(r+1)-1}\right)\right| .
$$

Note that by Definition 2.3 we obtain that

$$
A_{0}+B_{0}=2 .
$$

Finally, for every $i \in\{1, \ldots, k\}$ let $w_{i}:=\left|W_{i}\right|$; and let $\tilde{w}_{i}:=1$ if $w_{i} \geq 2$, and $\tilde{w}_{i}:=0$ if $w_{i}=1$. The two key lemmas below will help us to prove Theorem 1.1.

Key Lemma 1. For $r=1, \ldots, k$ we have the following.

(a) If $w_{r}=1$ then

$$
\begin{aligned}
A_{r}+B_{r} & \leq 2 \cdot A_{r-1}+B_{r-1} \\
A_{r} & \leq A_{r-1}+B_{r-1}, \quad \text { if } v_{f(r+1)} \text { is pleasant. }
\end{aligned}
$$

(b) If $w_{r} \geq 2$ then

$$
\begin{aligned}
A_{r}+B_{r} & \leq 2 \cdot F_{w_{r}} \cdot A_{r-1}+\left(F_{w_{r}+1}-1\right) \cdot B_{r-1} \\
A_{r} & \leq\left(F_{w_{r}}+F_{w_{r}-2}\right) \cdot A_{r-1}+F_{w_{r}} \cdot B_{r-1}, \quad \text { if } v_{f(r+1)} \text { is pleasant, }
\end{aligned}
$$

where $F_{i}$ denotes the ith Fibonacci number for $i \in \mathbb{N}_{0}$. (We assume that $F_{0}=0$ and $F_{1}=1$.)

(c) If $w_{r}=3$ and $v_{f(r+1)}$ is pleasant then additionally one of the following inequalities holds.

(c1) $A_{r} \leq 3 \cdot A_{r-1}+B_{r-1}$

(c2) $A_{r} \leq 2 \cdot A_{r-1}+2 \cdot B_{r-1}$

Key Lemma 2. Let $0 \leq p<q \leq k$ be integers such that for every $i$ with $p<i<q$ the vertex $v_{f(i+1)}$ is pleasant. If (6) - (9) and (c) hold then $A_{q}+B_{q} \leq 1.628^{\left(\sum_{i=p+1}^{q} w_{i}\right)}$. $\left(\frac{1.628}{2}\right)^{\left(\sum_{i=p+1}^{q} \tilde{w}_{i}\right)-1} \cdot\left(A_{p}+B_{p}\right)$. 
Proving Theorem 1.1 using Key Lemma 1 and Key Lemma 2 We now show that Key Lemma 1 and Key Lemma 2 imply Theorem 1.1. Let $u$ denote the number of unpleasant vertices in $\left\{v_{f(i)}: 2 \leq i \leq k\right\}$ and let $i_{1}<i_{2}<\ldots<i_{u}$ be the indices in $\{1, \ldots, k-1\}$ such that $v_{f\left(i_{1}+1\right)}, v_{f\left(i_{2}+1\right)}, \ldots, v_{f\left(i_{u}+1\right)}$ are unpleasant. Moreover, let $i_{0}:=0$ and $i_{u+1}:=k$. Note that for every $j \in\{0,1, \ldots, u\}$ we have that $v_{f(i+1)}$ is pleasant for every $i_{j}<i<i_{j+1}$. Hence, for every $j \in\{0,1, \ldots, u\}$ Key Lemma 2 gives that

$$
A_{i_{j+1}}+B_{i_{j+1}} \leq 1.6288^{\left(\sum_{i=i_{j}+1}^{i_{j+1}} w_{i}\right)} \cdot\left(\frac{1.628}{2}\right)^{\left(\sum_{i=i_{j}+1}^{i_{j+1}} \tilde{w}_{i}\right)-1} \cdot\left(A_{i_{j}}+B_{i_{j}}\right) .
$$

Recall that by Definition 4.1 we have that $f(k+1)=i_{\max }+1$. (10) together with (4) gives that

$$
\left|\operatorname{ch}\left(v_{i_{\max }}\right)\right|=A_{k}+B_{k} \leq 1.628^{\left(\sum_{i=1}^{k} w_{i}\right)} \cdot\left(\frac{1.628}{2}\right)^{\left(\sum_{i=1}^{k} \tilde{w}_{i}\right)-(u+1)} \cdot\left(A_{0}+B_{0}\right) .
$$

Recall that $a$ and $s$ denote the number of active vertices and outward patterns, respectively, in $\left\{v_{1}, \ldots, v_{i_{\max }}\right\}$. Since $W_{1}, \ldots, W_{k}$ is a partition of the active vertices in $v_{1}, \ldots, v_{i_{\max }}$ we get that $\sum_{i=1}^{k} w_{i}=a$ and $\sum_{i=1}^{k} \tilde{w}_{i}=s$. Moreover, we have that $u \leq u_{\text {tot }}$. Together with (5) and (11) this yields that

$$
\left|\operatorname{ch}\left(v_{i_{\max }}\right)\right| \leq 1.628^{a} \cdot\left(\frac{1.628}{2}\right)^{s-u_{\text {tot }}-1} \cdot 2 .
$$

If $i_{\max }=n-1$ then $a=\frac{n}{2}$ (by Remark 2.2) and $s=s_{\text {tot }}$, which together with (12), (2) and Observation 3.4 gives that $\mathcal{A}$ runs in time $1.628^{\frac{n}{2}} \cdot \operatorname{poly}(n)$, as claimed. If $i_{\max }<n-1$ we need one more argument. Since every outward pattern contains at least one active vertex we have that

$$
a^{\prime} \geq s^{\prime}
$$

Intuitively, for every outward pattern $P$ not contained in $\left\{v_{1}, \ldots, v_{i_{\max }}\right\}$ there is at least one active vertex not contained in $\left\{v_{1}, \ldots, v_{i_{\max }}\right\}$, hence for each such $P$ we save (in (12)) a factor of $1.628 \cdot \frac{1.628}{2}>1$. Formally, by (1) and (13) we get that $a=\frac{n}{2}-a^{\prime} \leq \frac{n}{2}-s^{\prime}$. Together with (12), (3) and (2) this gives that

$\left|\operatorname{ch}\left(v_{i_{\max }}\right)\right| \leq 1.628^{\frac{n}{2}-s^{\prime}} \cdot\left(\frac{1.628}{2}\right)^{s_{\mathrm{tot}}-s^{\prime}-u_{\mathrm{tot}}-2} \cdot 2 \leq 1.628^{\frac{n}{2}} \cdot\left(\frac{2}{1.628^{2}}\right)^{s^{\prime}} \cdot O(1) \leq 1.628^{\frac{n}{2}} \cdot O(1)$.

By Observation 3.4 this implies that $\mathcal{A}$ runs in time $1.628^{\frac{n}{2}} \cdot \operatorname{poly}(n)$, as claimed.

\section{Proof of Key Lemma 2}

\subsection{Some Auxiliary Lemmas}

We need some notation first. For $i \in \mathbb{N}$ we let $\mathbb{N}_{\geq i}$ and $\mathbb{N}_{\neq i}$ denote the set of natural numbers which are at least $i$, and the set of natural numbers which are different from $i$, 
respectively. For every matrix $M$ we let $M_{i j}$ denote the element in row $i$ and column $j$. We will consider the alphabet $\mathbb{A}=\mathbb{N}_{\neq 3} \cup\left\{3^{\prime}, 3^{\prime \prime}\right\}$.

Definition 5.1. For every $i \in \mathbb{A}$ with $i \notin\left\{1,3^{\prime}, 3^{\prime \prime}\right\}$ we let

$$
g(i):=\left(\begin{array}{cc}
F_{i}+F_{i-2} & F_{i} \\
F_{i-1} & F_{i-1}-1
\end{array}\right) .
$$

Moreover, we let

$$
g(1):=\left(\begin{array}{ll}
1 & 1 \\
1 & 0
\end{array}\right), \quad g\left(3^{\prime}\right):=\left(\begin{array}{ll}
3 & 1 \\
1 & 1
\end{array}\right), \quad g\left(3^{\prime \prime}\right):=\left(\begin{array}{ll}
2 & 2 \\
2 & 0
\end{array}\right) .
$$

We set $h(i):=g(i)_{11}+g(i)_{21}$ for every $i \in \mathbb{A}$.

Finally, let $s=\left(s_{1}, s_{2}, \ldots, s_{l}\right)$ be a sequence of elements of $\mathbb{A}$. We define $g(s):=$ $g\left(s_{1}\right) \cdot g\left(s_{2}\right) \cdots g\left(s_{l}\right)$, and $h(s):=g(s)_{11}+g(s)_{21}$. For the empty string $\varepsilon$ we define $g(\varepsilon)$ to be the identity matrix. Accordingly, $h(\varepsilon)=1$.

Suppose that for every $p+1 \leq r \leq q$ the corresponding inequalities of (6) - (9) and (c) are fulfilled with equality (the "corresponding inequalities" are (6) - (7) if $w_{r}=1$, (8) - (9) if $w_{r} \notin\{1,3\}$, and finally, (8) and one of $\{(\mathrm{c} 1),(\mathrm{c} 2)\}$ if $\left.w_{r}=3\right)$. If we interpret every $w_{r}$ with $w_{r}=3$ as $3^{\prime}$ if (c1) is fulfilled with equality, and as $3^{\prime \prime}$ otherwise, we get $\left(\begin{array}{l}A_{r} \\ B_{r}\end{array}\right)=g\left(w_{r}\right) \cdot\left(\begin{array}{l}A_{r-1} \\ B_{r-1}\end{array}\right)$, and thus $\left(\begin{array}{l}A_{q} \\ B_{q}\end{array}\right)=g\left(\left(w_{q}, w_{q-1}, \ldots, w_{p+1}\right)\right)\left(\begin{array}{l}A_{p} \\ B_{p}\end{array}\right)$. In the following we will bound $g\left(\left(w_{q}, w_{q-1}, \ldots, w_{p+1}\right)\right)$ and show that $A_{q}+B_{q}$ is at most $\left(A_{p}+B_{p}\right)$ times the sum of the elements in the first column of $g\left(\left(w_{q}, w_{q-1}, \ldots, w_{p+1}\right)\right)$. This will allow us to prove Key Lemma 2.

Bounding $g$ Unless otherwise indicated, by a sequence we always mean a sequence of elements of $\mathbb{A}$. The next two observations are a direct consequence of the definition of $g$.

Observation 5.2. Let $s=\left(s_{1}, \ldots, s_{l}\right)$ be a sequence and let $s^{\prime}=\left(s_{1}, \ldots, s_{i}\right)$, $s^{\prime \prime}=$ $\left(s_{i+1}, \ldots, s_{l}\right)$ for some $i \leq l$. Then $g(s)=g\left(s^{\prime}\right) g\left(s^{\prime \prime}\right)$.

Observation 5.3. For every $i \in \mathbb{A}$ we have $g(i)_{11} \geq g(i)_{12}$ and $g(i)_{21} \geq g(i)_{22}$. It follows by induction that for every sequence $s$ of length at least one we get $g(s)_{i 1} \geq g(s)_{i 2}$ for $i \in\{1,2\}$.

For a sequence $s=\left(s_{1}, \ldots, s_{l}\right)$ we let $\operatorname{sum}(s)$ and $\operatorname{num}_{\neq_{1}}(s)$ denote the sum of the $s_{i}$ (we interpret $3^{\prime}$ and $3^{\prime \prime}$ as 3 ), and the number of $s_{i}$ different from 1 , respectively. For instance, for $s=\left(2,3^{\prime}, 5,1,3^{\prime \prime}, 1\right)$ we have $\operatorname{sum}(s)=15$ and $\operatorname{num}_{\neq_{1}}(s)=4$. Finally, $s$ is called sufficient if

$$
h(s) \leq 1.628^{\text {sum }(s)} \cdot\left(\frac{1.628}{2}\right)^{\text {num }_{\neq 1}(s)-1},
$$

and insufficient otherwise. If (14) remains true when the right hand side is multiplied with $\frac{1.628}{2}$ (i.e., if $h(s) \leq 1.628^{\text {sum }(s)} \cdot\left(\frac{1.628}{2}\right)^{\text {num }_{\neq_{1}}(s)}$ ) then $s$ is called strong. 
We have $h(\varepsilon)=1=1.628^{0} \cdot\left(\frac{1.628}{2}\right)^{0}$, thus $\varepsilon$ is strong. It can be checked that the sequence $(i)$ is sufficient for every $i \in\left\{1,2,3^{\prime}, 3^{\prime \prime}, 4,5,6,7\right\}$. Moreover, due to the explicit formula for the Fibonacci numbers, we have for every $i \geq 8$,

$$
h(i)=2 F_{i} \leq \frac{2}{\sqrt{5}}\left(1.6181^{i}+0.6181^{i}\right) \leq 1.6181^{i}+0.6181^{8} \leq 1.03 \cdot 1.6181^{i} \leq 1.628^{i} .
$$

This directly implies the following.

Observation 5.4. Every sequence of length one is sufficient. Moreover, $\varepsilon$ is strong.

We now consider the concatenation of two sequences. Let $s=\left(s_{1}, \ldots, s_{l}\right)$ be a sequence and let $s^{\prime}=\left(s_{1}, \ldots, s_{i}\right), s^{\prime \prime}=\left(s_{i+1}, \ldots, s_{l}\right)$ for some $1 \leq i \leq l-1$. By Observation 5.2 and Observation 5.3 we have

$$
\begin{aligned}
h\left(s^{\prime}\right) h\left(s^{\prime \prime}\right) & =\left(g\left(s^{\prime}\right)_{11}+g\left(s^{\prime}\right)_{21}\right)\left(g\left(s^{\prime \prime}\right)_{11}+g\left(s^{\prime \prime}\right)_{21}\right) \\
& =g\left(s^{\prime}\right)_{11} g\left(s^{\prime \prime}\right)_{11}+g\left(s^{\prime}\right)_{11} g\left(s^{\prime \prime}\right)_{21}+g\left(s^{\prime}\right)_{21} g\left(s^{\prime \prime}\right)_{11}+g\left(s^{\prime}\right)_{21} g\left(s^{\prime \prime}\right)_{21} \\
& \geq g\left(s^{\prime}\right)_{11} g\left(s^{\prime \prime}\right)_{11}+g\left(s^{\prime}\right)_{12} g\left(s^{\prime \prime}\right)_{21}+g\left(s^{\prime}\right)_{21} g\left(s^{\prime \prime}\right)_{11}+g\left(s^{\prime}\right)_{22} g\left(s^{\prime \prime}\right)_{21} \\
& =\left(g\left(s^{\prime}\right) g\left(s^{\prime \prime}\right)\right)_{11}+\left(g\left(s^{\prime}\right) g\left(s^{\prime \prime}\right)\right)_{21} \\
& =g(s)_{11}+g(s)_{21}=h(s) .
\end{aligned}
$$

Observation 5.5. Let $s=\left(s_{1}, \ldots, s_{l}\right)$ be a sequence and let $s^{\prime}=\left(s_{1}, \ldots, s_{i}\right)$, $s^{\prime \prime}=$ $\left(s_{i+1}, \ldots, s_{l}\right)$ for some $1 \leq i \leq l-1$. Then $h(s) \leq h\left(s^{\prime}\right) h\left(s^{\prime \prime}\right)$. Moreover, by definition of $h(\varepsilon)$ we have $h(s)=h(\varepsilon) h(s)=h(s) h(\varepsilon)$.

It will turn out that sequences $s=\left(s_{1}, \ldots, s_{l}\right)$ containing at least one element $s_{i} \geq 50$ can be analyzed quite conveniently. So we first restrict on sequences over $\mathbb{A}^{\prime}:=\mathbb{A} \backslash \mathbb{N}_{\geq 50}$. Unless otherwise indicated, by a prefix of a sequence $s=\left(s_{1}, \ldots, s_{l}\right)$ we denote a sequence $s^{\prime}=\left(s_{1}, \ldots, s_{i}\right)$ with $1 \leq i \leq l$ (so the empty sequence $\varepsilon$ is not considered a prefix).

For every $i \in \mathbb{N}$ let $S_{\text {critical }}(i)$ denote the set of sequences of length $i$ over $\mathbb{A}^{\prime}$ where every prefix is sufficient but not strong. (Here it is crucial that $\varepsilon$ does not count as a prefix since otherwise by Observation 5.4 every sequence would contain a strong prefix.)

Note that for every $\left(s_{1}, \ldots, s_{i+1}\right) \in S_{\text {critical }}(i+1)$ we have $\left(s_{1}, \ldots, s_{i}\right) \in S_{\text {critical }}(i)$. In particular, if $S_{\text {critical }}(i)=\emptyset$ for some $i$ then $S_{\text {critical }}(j)=\emptyset$ for every $j \geq i$. We can obtain $S_{\text {critical }}(i+1)$ by considering every pair $\left(\left(s_{1}, \ldots, s_{i}\right), x\right) \in S_{\text {critical }}(i) \times \mathbb{A}^{\prime}$ and adding $\left(s_{1}, \ldots, s_{i}, x\right)$ to $S_{\text {critical }}(i+1)$ if and only if $\left(s_{1}, \ldots, s_{i}, x\right)$ is sufficient but not strong.

We use a computer program to determine $S_{\text {critical }}(i)$ for every $i, i=1, \ldots, 32$. A second computer program checks for every $i \in\{1, \ldots, 31\}$, every $\left(s_{1}, \ldots, s_{i}\right) \in S_{\text {critical }}(i)$ and every $x \in \mathbb{A}^{\prime}$ whether $\left(s_{1}, \ldots, s_{i}, x\right)$ is sufficient.

Observation 5.6. Our computer programs find that

(i) $S_{\text {critical }}(32)=\emptyset$, and

(ii) for every $i \in\{1, \ldots, 31\}$, every $\left(s_{1}, \ldots, s_{i}\right) \in S_{\text {critical }}(i)$ and every $x \in \mathbb{A}^{\prime}$ the sequence $\left(s_{1}, \ldots, s_{i}, x\right)$ is sufficient. 
By (i) we immediately get that $S_{\text {critical }}(j)=\emptyset$ for every $j \geq 32$.

Proposition 5.7. Every sequence over $\mathbb{A}^{\prime}$ is sufficient.

Proof: Suppose, for a contradiction, that there is an insufficient sequence $s=\left(s_{1}, \ldots, s_{l}\right)$ over $\mathbb{A}^{\prime}$ and let $s$ be the shortest sequence with this property. By Observation 5.4 we have $l \geq 2$. By minimality of $s$ every subsequence of $s$ of length at most $l-1$ is sufficient. If $s$ has a strong prefix $s^{\prime}=\left(s_{1}, \ldots, s_{i}\right)$ then by Observation 5.5 and insufficiency of $s$, we obtain for $s^{\prime \prime}:=\left(s_{i+1}, \ldots, s_{l}\right)$,

$$
\begin{aligned}
h\left(s^{\prime \prime}\right) \geq \frac{h(s)}{h\left(s^{\prime}\right)} & >1.628^{\text {sum }(s)} \cdot\left(\frac{1.628}{2}\right)^{\text {num }_{\neq_{1}}(s)-1} \cdot 1.628^{-\operatorname{sum}\left(s^{\prime}\right)} \cdot\left(\frac{1.628}{2}\right)^{- \text {num }_{\neq_{1}}\left(s^{\prime}\right)} \\
& =1.628^{\text {sum }\left(s^{\prime \prime}\right)} \cdot\left(\frac{1.628}{2}\right)^{\text {num }_{\neq_{1}}\left(s^{\prime \prime}\right)-1}
\end{aligned}
$$

implying that $s^{\prime \prime}$ is insufficient, which contradicts the minimality of $s$.

Hence $s$ does not have a strong prefix and therefore $\left(s_{1}, \ldots, s_{l-1}\right)$ does not have a strong prefix either. Moreover, by minimality of $s$, every prefix of $\left(s_{1}, \ldots, s_{l-1}\right)$ is sufficient. So $\left(s_{1}, \ldots, s_{l-1}\right) \in S_{\text {critical }}(l-1)$, and thus $S_{\text {critical }}(l-1) \neq \emptyset$. Observation 5.6.(i) implies that $l-1 \leq 31$. But then Observation 5.6.(ii) (for $i=l-1$ and $x=s_{l}$ ) yields that $s$ is sufficient, which leads to a contradiction.

We now generalize Proposition 5.7 to sequences over $\mathbb{A}$.

Proposition 5.8. Every sequence over $\mathbb{A}$ is sufficient.

Proof: We apply induction on the length of the sequences. For sequences of length at most one the claim holds due to Observation 5.4. So let $s=\left(s_{1}, \ldots, s_{l}\right)$ be a sequence with $l \geq 2$. If $s_{i} \in \mathbb{A}^{\prime}$ for every $i \in\{1, \ldots, l\}$ then $s$ is sufficient due to Proposition 5.7. Otherwise, $s_{i} \geq 50$ for some $i$, and by the explicit formula for the Fibonacci numbers we get

$$
h\left(s_{i}\right)=2 F_{s_{i}} \leq \frac{2}{\sqrt{5}}\left(1.6181^{s_{i}}+0.6181^{s_{i}}\right) \leq \frac{2}{\sqrt{5}} \cdot 1.001 \cdot 1.6181^{s_{i}} \leq 1.628^{s_{i}}\left(\frac{1.628}{2}\right)^{2} .
$$

That is, for $s:=\left(s_{i}\right)$, (14) remains true even when the right hand side is multiplied with $\left(\frac{1.628}{2}\right)^{2}$. Let $s^{\prime}=\left(s_{1}, \ldots, s_{i-1}\right)$ and $s^{\prime \prime}=\left(s_{i+1}, \ldots, s_{l}\right)$ (if $i=1$ or $i=l$ then $s^{\prime}$ or $s^{\prime \prime}$, respectively, is the empty sequence). Observation 5.5 gives that

$$
h(s) \leq h\left(s^{\prime}\right) h\left(s_{i}\right) h\left(s^{\prime \prime}\right) .
$$

By induction we get that

$$
h\left(s^{\prime}\right) h\left(s^{\prime \prime}\right) \leq 1.628^{\text {sum }\left(s^{\prime}\right)} \cdot\left(\frac{1.628}{2}\right)^{\text {num }_{\neq_{1}}\left(s^{\prime}\right)-1} \cdot 1.628^{\text {sum }\left(s^{\prime \prime}\right)} \cdot\left(\frac{1.628}{2}\right)^{\text {num }_{\neq 1}\left(s^{\prime \prime}\right)-1} .
$$


(15) - (17) imply that

$$
h(s) \leq 1.628^{\text {sum }(s)} \cdot\left(\frac{1.628}{2}\right)^{\text {num }_{\neq 1}(s)-1}
$$

as claimed.

The following is a direct consequence of Proposition 5.8.

Lemma 5.9. Let $\mathbb{A}=\mathbb{N}_{\neq 3} \cup\left\{3^{\prime}, 3^{\prime \prime}\right\}$ and let $g$, $h$ be defined as in Definition 5.1. For every sequence $s=\left(s_{1}, \ldots, s_{l}\right)$ over $\mathbb{A}$ we have $h(s) \leq 1.628^{\mathrm{sum}(s)} \cdot\left(\frac{1.628}{2}\right)^{\text {num }_{\neq_{1}}(s)-1}$.

\subsection{Derivation of Key Lemma 2}

Note that by assumption, for every $p+1 \leq r \leq q-1$ with $w_{r}=3$ either (c1) or (c2) is satisfied. For every $i \in\{p+1, \ldots, q\}$ we set

$$
s_{i}:= \begin{cases}w_{i}, & \text { if } w_{i} \neq 3 \\ 3^{\prime}, & \text { if } w_{i}=3 \text { and }(\mathrm{c} 1) \text { is satisfied } \\ 3^{\prime \prime}, & \text { if } w_{i}=3 \text { and }(\mathrm{c} 1) \text { is not satisfied }\end{cases}
$$

and $s:=\left(s_{q}, s_{q-1}, \ldots, s_{p+1}\right)$. Moreover, we define two sequences $\hat{A}_{p}, \ldots, \hat{A}_{q}$ and $\hat{B}_{p}, \ldots, \hat{B}_{q}$ by $\hat{A}_{p}:=A_{p}, \hat{B}_{p}:=B_{p}$, and for every $i \in\{p+1, \ldots, q\}$,

$$
\left(\begin{array}{c}
\hat{A}_{i} \\
\hat{B}_{i}
\end{array}\right)=g\left(s_{i}\right)\left(\begin{array}{c}
\hat{A}_{i-1} \\
\hat{B}_{i-1}
\end{array}\right)
$$

Note that

$$
\left(\begin{array}{c}
\hat{A}_{q} \\
\hat{B}_{q}
\end{array}\right)=g\left(s_{q}\right) \cdot g\left(s_{q-1}\right) \cdots g\left(s_{p+1}\right)\left(\begin{array}{c}
\hat{A}_{p} \\
\hat{B}_{p}
\end{array}\right)=g(s)\left(\begin{array}{c}
A_{p} \\
B_{p}
\end{array}\right)=\left(\begin{array}{c}
g(s)_{11} A_{p}+g(s)_{12} B_{p} \\
g(s)_{21} A_{p}+g(s)_{22} B_{p}
\end{array}\right),
$$

thus by Observation 5.3,

$$
\hat{A}_{q}+\hat{B}_{q} \leq g(s)_{11}\left(A_{p}+B_{p}\right)+g(s)_{21}\left(A_{p}+B_{p}\right)=h(s)\left(A_{p}+B_{p}\right) .
$$

We now show by induction that (i) $A_{i} \leq \hat{A}_{i}$ for every $p \leq i \leq q-1$, and (ii) $A_{i}+B_{i} \leq$ $\hat{A}_{i}+\hat{B}_{i}$ for every $p \leq i \leq q$. For $i=p$ the claim follows directly from the definition of $\hat{A}_{p}, \hat{B}_{p}$. We now let $p+1 \leq i \leq q-1$. By (6) - (9), (c) and induction we get

$$
\begin{aligned}
A_{i} & \leq g\left(s_{i}\right)_{11} \cdot A_{i-1}+g\left(s_{i}\right)_{12} \cdot B_{i-1} \\
& =g\left(s_{i}\right)_{12}\left(A_{i-1}+B_{i-1}\right)+\left(g\left(s_{i}\right)_{11}-g\left(s_{i}\right)_{12}\right) A_{i-1} \\
& \leq g\left(s_{i}\right)_{12}\left(\hat{A}_{i-1}+\hat{B}_{i-1}\right)+\left(g\left(s_{i}\right)_{11}-g\left(s_{i}\right)_{12}\right) \hat{A}_{i-1}=\hat{A}_{i} .
\end{aligned}
$$

Note that Observation 5.3 guarantees that on the second line, $A_{i-1}$ is multiplied with a positive factor, which is crucial for the inequality on the third line. Finally, for $p+1 \leq i \leq q$ 
we similarly get

$$
\begin{aligned}
A_{i}+B_{i} & \leq\left(g\left(s_{i}\right)_{11}+g\left(s_{i}\right)_{21}\right) A_{i-1}+\left(g\left(s_{i}\right)_{12}+g\left(s_{i}\right)_{22}\right) B_{i-1} \\
& =\left(g\left(s_{i}\right)_{12}+g\left(s_{i}\right)_{22}\right)\left(A_{i-1}+B_{i-1}\right)+\left(g\left(s_{i}\right)_{11}+g\left(s_{i}\right)_{21}-g\left(s_{i}\right)_{12}-g\left(s_{i}\right)_{22}\right) A_{i-1} \\
& \leq\left(g\left(s_{i}\right)_{12}+g\left(s_{i}\right)_{22}\right)\left(\hat{A}_{i-1}+\hat{B}_{i-1}\right)+\left(g\left(s_{i}\right)_{11}+g\left(s_{i}\right)_{21}-g\left(s_{i}\right)_{12}-g\left(s_{i}\right)_{22}\right) \hat{A}_{i-1} \\
& =\hat{A}_{i}+\hat{B}_{i} .
\end{aligned}
$$

Hence,

$$
A_{q}+B_{q} \leq \hat{A}_{q}+\hat{B}_{q}
$$

Lemma 5.9, (18) and (19) imply that

$$
A_{q}+B_{q} \leq h(s)\left(A_{p}+B_{p}\right) \leq 1.628^{\operatorname{sum}(s)} \cdot\left(\frac{1.628}{2}\right)^{\text {num }_{\neq 1}(s)-1}\left(A_{p}+B_{p}\right) .
$$

Note that $\operatorname{sum}(s)=\sum_{i=p+1}^{q} w_{i}$ and $\operatorname{num}_{\neq 1}(s)=\sum_{i=p+1}^{q} \tilde{w}_{i}$. Together with (20) this implies Key Lemma 2.

\section{Proof of Key Lemma 1}

\subsection{Some Auxiliary Lemmas}

Before proving Key Lemma 1 we state some auxiliary lemmas. We first give some basic properties of the vertices and choices we consider. Recall that whenever we process a passive vertex $v_{i}$ in $P_{\text {ham }}$ we have at most one option to decide whether or not to select the outgoing edge of $v_{i}$. So we obtain the following.

Observation 6.1. Let $j \leq l$ such that $v_{j+1}, \ldots, v_{l}$ are all passive. Then every $C \in \operatorname{ch}\left(v_{j}\right)$ has at most one extension in $\operatorname{ch}\left(v_{l}\right)$. In particular, for every $i \leq j$ and every $D \in \operatorname{ch}\left(v_{i}\right)$ we have

$$
\left|\operatorname{ch}_{\mid D}\left(v_{l}\right)\right| \leq\left|\operatorname{ch}_{\mid D}\left(v_{j}\right)\right| .
$$

We now evaluate Observation 6.1 for the case where $l=f(r+1)-1$ for some $1 \leq r \leq k$.

Observation 6.2. Let $1 \leq r \leq k$. By assumption, $v_{l(r)+1}, \ldots, v_{f(r+1)-1}$ are all passive. Let $l(r) \leq j \leq f(r+1)-1$. Then every $C \in \operatorname{ch}\left(v_{j}\right)$ has at most one extension in $\operatorname{ch}\left(v_{f(r+1)-1}\right)$. In particular, for every $D \in \operatorname{ch}\left(v_{f(r)-1}\right)$,

$$
\left|\mathrm{ch}_{\mid D}\left(v_{f(r+1)-1}\right)\right| \leq\left|\mathrm{ch}_{\mid D}\left(v_{l(r)}\right)\right| .
$$

Figure 5 illustrates Observation 6.2. By Observation 3.1 we have the following.

Observation 6.3. Let $i_{\text {start }}<i_{\text {end }}$ be integers where $v_{i_{\text {start }}+1}, v_{i_{\text {start }}+2}, \ldots, v_{i_{\text {end }}-1}$ are all passive and let $C \in \operatorname{ch}\left(v_{j}\right)$ for some $j \geq i_{\text {end }}-1$ such that for every $i \in\left\{i_{\text {start }}+1, \ldots, i_{\text {end }}-\right.$ $1\}$ the incoming diagonal of $v_{i}$ belongs to $C$. Then for every $i \in\left\{i_{\text {start }}+1, \ldots, i_{\text {end }}-1\right\}$ we have $\left(v_{i-1}, v_{i}\right) \in C \Leftrightarrow\left(v_{i}, v_{i+1}\right) \notin C$. In particular,

$$
\begin{array}{lll}
\left(v_{i_{\text {start }}}, v_{i_{\text {start }}+1}\right) \in C \Leftrightarrow\left(v_{i_{\text {end }}-1}, v_{i_{\text {end }}}\right) \in C, & \text { if } i_{\text {end }}-i_{\text {start }} \equiv 1 & (\bmod 2) \\
\left(v_{i_{\text {start }}}, v_{i_{\text {start }}+1}\right) \in C \Leftrightarrow\left(v_{i_{\text {end }}-1}, v_{i_{\text {end }}}\right) \notin C, & \text { if } i_{\text {end }}-i_{\text {start }} \equiv 0 & (\bmod 2)
\end{array}
$$




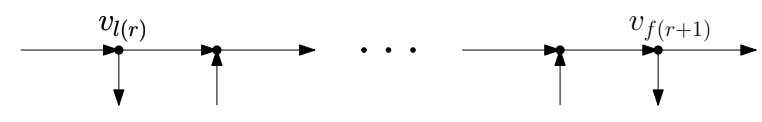

Figure 5: Every vertex $v_{i}$ with $l(r)<i<f(r+1)$ is passive. Thus, for $l(r) \leq j \leq$ $f(r+1)-1$, every choice $C \in \operatorname{ch}\left(v_{j}\right)$ has at most one extension in $\operatorname{ch}\left(v_{f(r+1)-1}\right)$.

Since we will often deal with extensions containing a particular edge the next definition will be helpful.

Definition. Let $i \leq j$. For every choice $D \in \operatorname{ch}\left(v_{i}\right)$ and every edge e we let $\operatorname{ch}_{\mid D,+e}\left(v_{j}\right)$ $\left(\mathrm{ch}_{\mid D,-e}\left(v_{j}\right)\right.$, respectively) denote the elements of $\mathrm{ch}_{\mid D}\left(v_{j}\right)$ which contain (do not contain, respectively) e. We sometimes abbreviate $\mathrm{ch}_{\mid D,+\left(v_{j}, v_{j+1}\right)}\left(v_{j}\right)$ and $\mathrm{ch}_{\mid D,-\left(v_{j}, v_{j+1}\right)}\left(v_{j}\right)$ by $\operatorname{ch}_{\mid D}^{\text {sel }}\left(v_{j}\right)$ and $\operatorname{ch}_{\mid D}^{\text {unsel }}\left(v_{j}\right)$, respectively.

The next proposition is a consequence of Observation 3.1.

Proposition 6.4. Let $i \leq j$ such that $v_{i}$ is an active vertex, let d denote the outgoing diagonal of $v_{i}$ and let $C, C^{\prime} \in \operatorname{ch}\left(v_{i-1}\right)$ where $\left(v_{i-1}, v_{i}\right) \in C$ and $\left(v_{i-1}, v_{i}\right) \notin C^{\prime}$. Moreover, let $D^{\prime}=C \cup\left\{\left(v_{i}, v_{i+1}\right)\right\}, D^{\prime \prime}=C \cup\{d\}$ and $E=C^{\prime} \cup\left\{\left(v_{i}, v_{i+1}\right), d\right\}$. Then $(i) \operatorname{ch}_{\mid C}\left(v_{j}\right)=$ $\operatorname{ch}_{\mid D^{\prime}}\left(v_{j}\right) \cup \operatorname{ch}_{\mid D^{\prime \prime}}\left(v_{j}\right)$, and (ii) $\operatorname{ch}_{\mid C^{\prime}}\left(v_{j}\right)=\operatorname{ch}_{\mid E}\left(v_{j}\right)$.

Proof: Suppose first that we process $v_{i}$ in $P_{\text {ham }}$ after having selected the edges in $C^{\prime}$. Then we are forced to select both $\left(v_{i}, v_{i+1}\right)$ and $d$, which implies (ii). Suppose now that we process $v_{i}$ in $P_{\text {ham }}$ after having selected the edges in $C$. Then we have two options: We can select either $\left(v_{i}, v_{i+1}\right)$ or $d$. This shows (i).

Proposition 6.4 (for $j=i$ ) directly implies the following.

Corollary 6.5. Let $C \in \operatorname{ch}\left(v_{i-1}\right)$. Then $\left|\operatorname{ch}_{\mid C}^{\text {sel }}\left(v_{i}\right)\right|,\left|\operatorname{ch}_{\mid C}^{\text {unsel }}\left(v_{i}\right)\right| \leq 1$.

By Observation 3.3 we can obtain $\left|\operatorname{ch}\left(v_{f(r+1)-1}\right)\right|$ by summing up $\left|\operatorname{ch}_{\mid C}\left(v_{f(r+1)-1}\right)\right|$ over each choice $C \in \operatorname{ch}\left(v_{l(r)-1}\right)$. The next proposition bounds the contribution of each choice $C \in \operatorname{ch}\left(v_{l(r)-1}\right)$ to this sum.

Proposition 6.6. Let $1 \leq r \leq k$ and let $C, C^{\prime} \in \operatorname{ch}\left(v_{l(r)-1}\right)$ where $\left(v_{l(r)-1}, v_{l(r)}\right) \in C$ and $\left(v_{l(r)-1}, v_{l(r)}\right) \notin C^{\prime}$. Then $(i) C^{\prime}$ has at most one extension in $\mathrm{ch}\left(v_{f(r+1)-1}\right)$, and (ii) $C$ has at most two extensions in $\mathrm{ch}\left(v_{f(r+1)-1}\right)$, at most one of which contains $\left(v_{l(r)}, v_{l(r)+1}\right)$ and at most one of which does not contain $\left(v_{l(r)}, v_{l(r)+1}\right)$.

Proof: By Proposition 6.4 (for $i=j=l(r)$ ) and Corollary 6.5 (recall that by definition $\operatorname{ch}_{\mid C}^{\text {sel }}\left(v_{i}\right)=\operatorname{ch}_{\mid C,+\left(v_{i}, v_{i+1}\right)}\left(v_{i}\right)$ and $\left.\operatorname{ch}_{\mid C}^{\text {unsel }}\left(v_{i}\right)=\operatorname{ch}_{\mid C,-\left(v_{i}, v_{i+1}\right)}\left(v_{i}\right)\right)$ we get

$$
\begin{aligned}
& \left|\operatorname{ch}_{\mid C^{\prime}}\left(v_{l(r)}\right)\right| \leq 1, \\
& \left|\operatorname{ch}_{\mid C}\left(v_{l(r)}\right)\right| \leq 2, \\
& \left|\operatorname{ch}_{\mid C,+\left(v_{l(r)}, v_{l(r)+1}\right)}\left(v_{l(r)}\right)\right|, \quad\left|\operatorname{ch}_{\mid C,-\left(v_{l(r)}, v_{l(r)+1}\right)}\left(v_{l(r)}\right)\right| \leq 1 .
\end{aligned}
$$

Observation 6.2 (for $j=l(r)$ ) yields that $(21)$ - (23) remain true when " $\left(v_{l(r)}\right)$ " is replaced with " $\left(v_{f(r+1)-1}\right)$ ", which concludes the proof.

As we will point out later, Proposition 6.6 implies (6). 


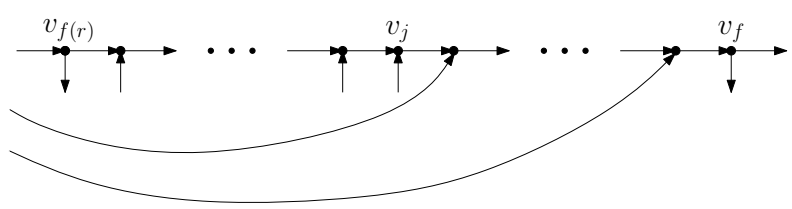

(a) Case 1: $f(r)=l(r)$. Then $i=f(r)$.

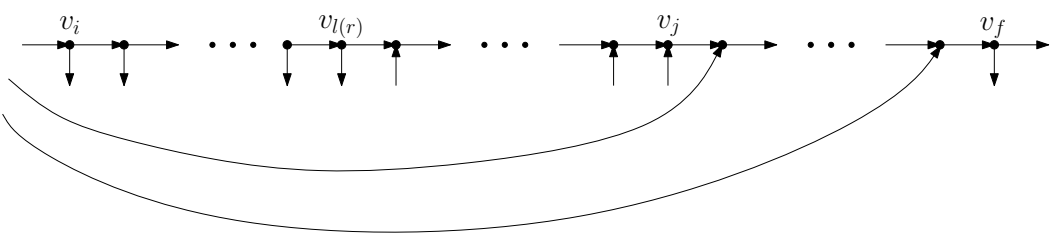

(b) Case 2: $f(r)<l(r)$. In this illustration we have $i<l(r)$. However, it is also possible that $i=l(r)$.

Figure 6: According to our assumption every incoming diagonal of a vertex in $\left\{v_{j+1}, \ldots, v_{f-1}\right\}$ has its source in $\left\{v_{1}, \ldots, v_{i-1}\right\}$. The above figures illustrate the situation for the case where $f(r)=l(r)$ and for the case where $f(r)<l(r)$. (In both figures we have $j>l(r)$ but it is also possible that $j=l(r)$.)

Bounding the number of choices containing a certain edge Let $1 \leq r \leq k$ such that $v_{f(r+1)}$ is pleasant. We now bound $\left|\mathrm{ch}^{\text {sel }}\left(v_{f(r+1)-1}\right)\right|$. This will help us to prove (7), (9) and (c). Let $f:=f(r+1)$, and let $f(r) \leq i \leq l(r) \leq j \leq f-1$ such that every incoming diagonal of a vertex in $\left\{v_{j+1}, \ldots, v_{f-1}\right\}$ has its source in $\left\{v_{1}, \ldots, v_{i-1}\right\}$. The situation is depicted in Figure 6. We fix two choices $C \in \operatorname{ch}\left(v_{i-1}\right)$ and $D \in \operatorname{ch}_{\mid C}^{\text {sel }}\left(v_{f-1}\right)$.

Observation 6.7. By definition of $l(r)$ and $f$, the vertices $v_{j+1}, \ldots, v_{f-1}$ are all passive.

We remark that choosing $i, j:=l(r)$ satisfies the condition that every incoming diagonal of a vertex in $\left\{v_{j+1}, \ldots, v_{f-1}\right\}$ has its source in $\left\{v_{1}, \ldots, v_{i-1}\right\}$. (Indeed, by assumption $v_{f}$ is pleasant and thus every incoming diagonal of a vertex in $\left\{v_{l(r)+1}, \ldots, v_{f-1}\right\}$ has its source in $\left\{v_{1}, \ldots, v_{l(r)-1}\right\}$.) Our goal is to show that many properties of $D$ are uniquely determined by $C$.

Observation 6.8. For every incoming diagonal d of a vertex in $\left\{v_{j+1}, \ldots, v_{f-1}\right\}$ it holds that $d \in D$ if and only if $d \in C$.

We let $s:=f-j$ and

$$
m:=\max _{x \in\{1 \ldots s\}}\left(x: \text { for } l=1 \ldots x-1 \text { the incoming diagonal of } v_{j+l} \text { belongs to } C\right)
$$

If $m<s$ then the incoming diagonal of $v_{j+m}$ does not belong to $C$ and therefore by Observation 3.1 and Observation 6.8 the edge $\left(v_{j+m-1}, v_{j+m}\right)$ is in $D$. Otherwise, $v_{j+m}=$ 


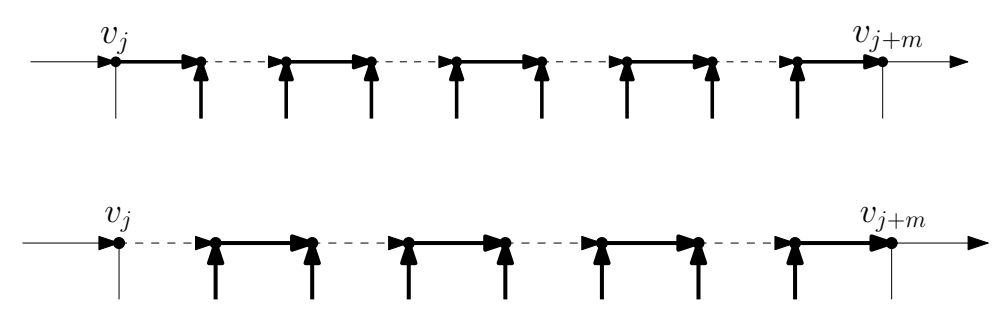

Figure 7: An illustration of (26) for the case where $m$ is odd (on the top) and the case where $m$ is even (on the bottom). The edges of $D$ are drawn thick, the edges not belonging to $D$ are drawn dashed. A solid edge may or may not belong to $D$. A diagonal is drawn undirected if it might be oriented either way.

$v_{f}$ and the incoming diagonals of $v_{j+1}, \ldots, v_{f-1}$ all belong to $C$. Moreover, by assumption, $\left(v_{f-1}, v_{f}\right)=\left(v_{j+m-1}, v_{j+m}\right)$ belongs to $D$. So in either case we have

$$
\left(v_{j+m-1}, v_{j+m}\right) \in D
$$

We now determine whether $\left(v_{j}, v_{j+1}\right)$ belongs to $D$. By Observation 6.8 and (24) we have that the incoming diagonals of $v_{j+1}, \ldots, v_{j+m-1}$ all belong to $D$. Observation 6.3 (for $i_{\text {start }}=j$ and $i_{\text {end }}=j+m$ ) and (25) yield that

$$
\left(v_{j}, v_{j+1}\right) \in D \Leftrightarrow m \equiv 1 \quad(\bmod 2) .
$$

Figure 7 illustrates (26). Note that $m$ can be considered as a function of $C$. Considering the function $g(C):=1$ if $m \equiv 1(\bmod 2)$, and $g(C):=0$ otherwise, we obtain the following.

Proposition 6.9. Let $1 \leq r \leq k$ such that $v_{f(r+1)}$ is pleasant and let $f(r) \leq i \leq l(r) \leq$ $j \leq f(r+1)-1$ such that every incoming diagonal of a vertex in $\left\{v_{j+1}, \ldots, v_{f(r+1)-1}\right\}$ has its source in $\left\{v_{1}, \ldots, v_{i-1}\right\}$. Moreover, let $C \in \operatorname{ch}\left(v_{i-1}\right)$. There is a value $g(C) \in\{0,1\}$ such that every extension $D \in \mathrm{ch}_{\mid C}^{\text {sel }}\left(v_{f(r+1)-1}\right)$ has the property that

$$
\left(v_{j}, v_{j+1}\right) \in D \Leftrightarrow g(C)=1 .
$$

In particular,

$$
\left|\operatorname{ch}_{\mid C}^{\text {sel }}\left(v_{f(r+1)-1}\right)\right| \leq \max _{\oplus \in\{+,-\}}\left(\left|\operatorname{ch}_{\mid C, \oplus\left(v_{j}, v_{j+1}\right)}\left(v_{f(r+1)-1}\right)\right|\right) .
$$

Suppose that $v_{f(r+1)}$ is pleasant. Applying Proposition 6.9 for $i=j=l(r)$ then gives that $\left|\operatorname{ch}_{\mid C}^{\text {sel }}\left(v_{f(r+1)-1}\right)\right| \leq \max _{\oplus \in\{+,-\}}\left(\left|\operatorname{ch}_{\mid C, \oplus\left(v_{l(r)}, v_{l(r)+1}\right)}\left(v_{f(r+1)-1}\right)\right|\right)$. Together with Proposition 6.6 this implies the next corollary.

Corollary 6.10. Let $1 \leq r \leq k$ such that $v_{f(r+1)}$ is pleasant and let $C \in \operatorname{ch}\left(v_{l(r)-1}\right)$. Then $\left|\operatorname{ch}_{\mid C}^{\text {sel }}\left(v_{f(r+1)-1}\right)\right| \leq 1$.

As we will point out later, Corollary 6.10 implies (7). 
Bounding the number of choices for vertices in a pattern We now derive some auxiliary propositions which will help us to show (8) and (9). The next proposition bounds the number of choices for sequences of active vertices, which in particular occur in patterns.

Proposition 6.11. Let $i \geq 0$ and let $x$ be such that $v_{x+1}, \ldots, v_{x+i}$ are all active. For every choice $C \in \operatorname{ch}\left(v_{x}\right)$ we have $\left|\operatorname{ch}_{\mid C}\left(v_{x+i}\right)\right| \leq \begin{cases}F_{i+2}, & \text { if }\left(v_{x}, v_{x+1}\right) \in C \\ F_{i+1}, & \text { if }\left(v_{x}, v_{x+1}\right) \notin C\end{cases}$

Proof: We apply induction. The claim is clearly true for $i=0$. Now let $i \geq 1$ and let $d$ denote the outgoing diagonal of $v_{x+1}$. We first consider the case where $\left(v_{x}, v_{x+1}\right) \notin C$. Let $D=C \cup\left\{\left(v_{x+1}, v_{x+2}\right), d\right\}$. By Proposition 6.4 and induction we get $\left|\operatorname{ch}_{\mid C}\left(v_{x+i}\right)\right| \leq$ $\left|\mathrm{ch}_{\mid D}\left(v_{x+i}\right)\right| \leq F_{i+1}$, as claimed.

We now consider the case where $\left(v_{x}, v_{x+1}\right) \in C$. Let $D^{\prime}=C \cup\left\{\left(v_{x+1}, v_{x+2}\right)\right\}$ and let $D^{\prime \prime}=C \cup\{d\}$. By Proposition 6.4 we have

$$
\left|\operatorname{ch}_{\mid C}\left(v_{x+i}\right)\right| \leq\left|\operatorname{ch}_{\mid D^{\prime}}\left(v_{x+i}\right)\right|+\left|\operatorname{ch}_{\mid D^{\prime \prime}}\left(v_{x+i}\right)\right| .
$$

By induction we obtain $\left|\operatorname{ch}_{\mid D^{\prime}}\left(v_{x+i}\right)\right| \leq F_{i+1}$ and $\left|\operatorname{ch}_{\mid D^{\prime \prime}}\left(v_{x+i}\right)\right| \leq F_{i}$. Hence $\left|\operatorname{ch}_{\mid C}\left(v_{x+i}\right)\right| \leq$ $F_{i+1}+F_{i}=F_{i+2}$.

We will also need the following slight modification of Proposition 6.11. For every choice $C \in \operatorname{ch}\left(v_{x}\right)$ and every $i \geq 1$ we let $S(C, i)$ denote the set of choices $D \in \operatorname{ch}_{C}\left(v_{x+i}\right)$ where for some $j \in\{1, \ldots, i\}$ the edge $\left(v_{x+j}, v_{x+j+1}\right)$ does not belong to $D$.

Proposition 6.12. Let $i \geq 1$ and let $x$ be such that $v_{x+1}, \ldots, v_{x+i}$ are all active. For every $C \in \operatorname{ch}\left(v_{x}\right)$ we have $|S(C, i)| \leq \begin{cases}F_{i+2}-1, & \text { if }\left(v_{x}, v_{x+1}\right) \in C \\ F_{i+1}-1, & \text { if }\left(v_{x}, v_{x+1}\right) \notin C\end{cases}$

Proof: We apply induction. The claim is clearly true for $i=1$. So let $i \geq 2$ and let $d$ denote the outgoing diagonal of $v_{x+1}$. We first consider the case where $\left(v_{x}, v_{x+1}\right) \notin C$. Let $D=C \cup\left\{\left(v_{x+1}, v_{x+2}\right), d\right\} . S(C, i)$ consists of all choices $E \in \operatorname{ch}_{\mid D}\left(v_{x+i}\right)$ where for some $j \in\{1, \ldots, i-1\}$ the edge $\left(v_{x+1+j}, v_{x+2+j}\right)$ does not belong to $E$. Hence by induction, $|S(C, i)| \leq F_{(i-1)+2}-1=F_{i+1}-1$. We now consider the case where $\left(v_{x+1}, v_{x+2}\right) \in C$. Let $D^{\prime}=C \cup\left\{\left(v_{x+1}, v_{x+2}\right)\right\}$ and let $D^{\prime \prime}=C \cup\{d\}$. Note that no element in $D^{\prime \prime}$ contains $\left(v_{x+1}, v_{x+2}\right)$, thus every element in $\operatorname{ch}_{\mid D^{\prime \prime}}\left(v_{x+i}\right)$ belongs to $S(C, i)$. By induction and by Proposition 6.11 we obtain that $|S(C, i)| \leq \mid \operatorname{ch}_{\mid D^{\prime \prime}}\left(v_{x+i}\right)+F_{(i-1)+2}-1 \leq F_{i}+F_{i+1}-1=$ $F_{i+2}-1$, as claimed.

We now bound the number of choices for vertices forming a pattern. Recall that here we use "pattern" as an abbreviation for "outward pattern". By Definition 2.5 and by construction of the $W_{i}$ we obtain the following.

Observation 6.13. Let $1 \leq r \leq k$ where $w_{r} \geq 2$ and let $m=w_{r}$. Then $W_{r}=$ $\left\{v_{f(r)}, \ldots, v_{f(r)+m-1}\right\}$ and the sequence $\left(v_{f(r)}, \ldots, v_{f(r)+m}\right)$ forms a pattern. In particular, $v_{f(r)}, \ldots, v_{f(r)+m-1}$ are all active and $\left(v_{f(r)}, v_{f(r)+m}\right)$ is the outgoing diagonal of $v_{f(r)}$.

Figure 8 illustrates Observation 6.13. 


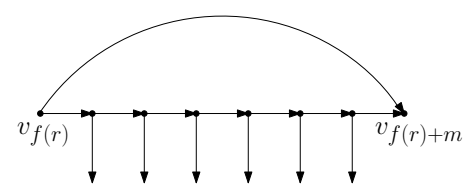

Figure 8: A pattern.

Proposition 6.14. Let $1 \leq r \leq k$ where $w_{r} \geq 2$ and let $m=w_{r}$. Moreover, let $C, C^{\prime} \in \operatorname{ch}\left(v_{f(r)-1}\right)$ where $\left(v_{f(r)-1}, v_{f(r)}\right) \in C$ and $\left(v_{f(r)-1}, v_{f(r)}\right) \notin C^{\prime}$. We have

(a) $\left|\operatorname{ch}_{\mid C}\left(v_{f(r+1)-1}\right)\right| \leq 2 F_{m}$,

(b) $\left|\mathrm{ch}_{\mid C^{\prime}}\left(v_{f(r+1)-1}\right)\right| \leq F_{m+1}-1$,

(c) if $v_{f(r+1)}$ is pleasant then $\left|\mathrm{ch}_{\mid C}^{\mathrm{sel}}\left(v_{f(r+1)-1}\right)\right| \leq F_{m+1}$,

(d) if $v_{f(r+1)}$ is pleasant then $\left|\operatorname{ch}_{\mid C^{\prime}}^{\text {sel }}\left(v_{f(r+1)-1}\right)\right| \leq F_{m}$.

Proof: Let $f:=f(r)$ and $f^{\prime}:=f(r+1)$. We first show (c) and (d). To this end we assume that $v_{f(r+1)}$ is pleasant. By Proposition 6.11 (for $x=f-1$ and $i=m-1$ ) we have $\left|\operatorname{ch}_{\mid C}\left(v_{f+m-2}\right)\right| \leq F_{m+1}$. Let $D \in \operatorname{ch}_{\mid C}\left(v_{f+m-2}\right)$. Note that $f+m-2=l(r)-1$, thus Corollary 6.10 gives that $\left|\operatorname{ch}_{\mid D}^{\text {sel }}\left(v_{f^{\prime}-1}\right)\right| \leq 1$. By Observation 3.3 we thus get $\left|\operatorname{ch}_{\mid C}^{\text {sel }}\left(v_{f^{\prime}-1}\right)\right| \leq$ $\sum_{D \in \mathrm{ch}_{\mid C}\left(v_{f+m-2}\right)}\left|\operatorname{ch}_{\mid D}^{\text {sel }}\left(v_{f^{\prime}-1}\right)\right| \leq\left|\operatorname{ch}_{\mid C}\left(v_{f+m-2}\right)\right| \leq F_{m+1}$, which implies (c). Similarly, we get $\left|\operatorname{ch}_{\mid C^{\prime}}^{\text {sel }}\left(v_{f^{\prime}-1}\right)\right| \leq\left|\operatorname{ch}_{\mid C^{\prime}}\left(v_{f+m-2}\right)\right| \leq F_{m}$, which implies (d).

We now show (b). Let $D \in \operatorname{ch}_{\mid C^{\prime}}\left(v_{f^{\prime}-1}\right)$. By Observation 3.1 the edge $\left(v_{f}, v_{f+m}\right)$ belongs to $D$ and therefore (also by Observation 3.1), at least one edge of the path $v_{f}, v_{f+1}, \ldots, v_{f+m}$ is not contained in $D$. Together with Observation 6.2 this implies that $\left|\mathrm{ch}_{\mid C^{\prime}}\left(v_{f^{\prime}-1}\right)\right|$ is bounded by the number of choices $D \in \mathrm{ch}_{\mid C^{\prime}}\left(v_{f+m-1}\right)$ where for some $1 \leq j \leq m$ the edge $\left(v_{f-1+j}, v_{f-1+j+1}\right)$ does not belong to $D$. By Proposition 6.12 (for $x=f-1$ and $i=m)$ this is at most $F_{m+1}-1$. This shows (b).

Finally, we prove (a). Let $D^{\prime}=C \cup\left\{\left(v_{f}, v_{f+1}\right)\right\}$ and $D^{\prime \prime}=C \cup\left\{\left(v_{f}, v_{f+m}\right)\right\}$. Figure 9 shows an illustration of $D^{\prime}$ and $D^{\prime \prime}$. Observation 6.2 and Proposition 6.4 give that

$$
\left|\operatorname{ch}_{\mid C}\left(v_{f^{\prime}-1}\right)\right| \leq\left|\operatorname{ch}_{\mid C}\left(v_{f+m-1}\right)\right| \leq\left|\operatorname{ch}_{\mid D^{\prime}}\left(v_{f+m-1}\right)\right|+\left|\operatorname{ch}_{\mid D^{\prime \prime}}\left(v_{f+m-1}\right)\right| .
$$

By Proposition 6.11 we have

$$
\left|\operatorname{ch}_{\mid D^{\prime \prime}}\left(v_{f+m-1}\right)\right| \leq F_{m},
$$

By Observation 3.1 and the fact that $\left(v_{f}, v_{f}+m\right)$ does not belong to $D^{\prime}$ we obtain that every choice of $\mathrm{ch}_{\mid D^{\prime}}\left(v_{f+m-1}\right)$ contains $\left(v_{f+m-1}, v_{f+m}\right)$. Together with Observation 3.3, Corollary 6.5 and Proposition 6.11 this gives that $\left|\mathrm{ch}_{\mid D^{\prime}}\left(v_{f+m-1}\right)\right| \leq\left|\operatorname{ch}_{\mid D^{\prime}}^{\text {sel }}\left(v_{f+m-1}\right)\right| \leq$ $\sum_{E \in \mathrm{ch}_{D^{\prime}}\left(v_{f+m-2}\right)}\left|\operatorname{ch}_{\mid E}^{\mathrm{sel}}\left(v_{f+m-1}\right)\right| \leq\left|\mathrm{ch}_{D^{\prime}}\left(v_{f+m-2}\right)\right| \leq F_{m}$. Together with (27) and (28) this implies (a).

In the sequel we will point out that Proposition 6.14.(a) and 6.14.(b) directly imply (8) and that Proposition 6.14.(c) and 6.14.(d) directly imply a weaker version of (9) where 

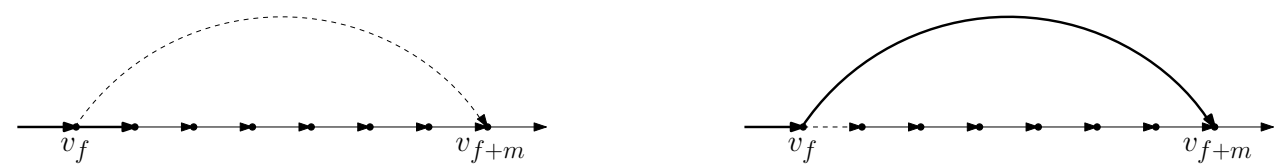

Figure 9: An illustration of $D^{\prime}$ (on the left) and $D^{\prime \prime}$ (on the right).

the coefficient $\left(F_{w_{r}}+F_{w_{r}-2}\right)$ is replaced with $F_{w_{r}+1}$. We will also sketch how Proposition 6.6, Corollary 6.10 and Proposition 6.14 together with a slightly modified version of Key Lemma 2 allow us to prove a weaker version of Theorem 1.1 where " 1.64 " is replaced with "1.628".

In the next subsection we improve Proposition 6.14.(c) for every $w_{r} \neq 3$, and Proposition 6.14.(d) for $w_{r}=3$.

\subsection{Refining Proposition 6.14}

We will use the following adaptation of Proposition 6.11.

Proposition 6.15. Let $i \geq 1$ and let $x$ be such that $v_{x+1}, \ldots, v_{x+i}$ are all active, and let $C \in \operatorname{ch}\left(v_{x}\right)$. Then $\left|\operatorname{ch}_{\mid C}^{\mathrm{sel}}\left(v_{x+i}\right)\right| \leq \begin{cases}F_{i+1}, & \text { if }\left(v_{x}, v_{x+1}\right) \in C \\ F_{i}, & \text { if }\left(v_{x}, v_{x+1}\right) \notin C\end{cases}$ and $\left|\operatorname{ch}_{\mid C}^{\text {unsel }}\left(v_{x+i}\right)\right| \leq \begin{cases}F_{i}, & \text { if }\left(v_{x}, v_{x+1}\right) \in C \\ F_{i-1}, & \text { if }\left(v_{x}, v_{x+1}\right) \notin C\end{cases}$

Proof: We apply induction. Corollary 6.5 implies that $\left|\operatorname{ch}_{\mid C}^{s}\left(v_{x+1}\right)\right| \leq 1$ for $\mathrm{s} \in\{$ sel, unsel $\}$, and Observation 3.1 gives that $\left|\mathrm{ch}_{\mid C}^{\text {unsel }}\left(v_{x+1}\right)\right|=0$ if $\left(v_{x}, v_{x+1}\right) \notin C$. So the claim holds for $i=1$. Let $i \geq 2$ and let $d$ denote the outgoing diagonal of $v_{x+1}$. We first consider the case where $\left(v_{x}, v_{x+1}\right) \notin C$. Let $D=C \cup\left\{\left(v_{x+1}, v_{x+2}\right), d\right\}$. By Proposition 6.4 and induction we get that $\left|\operatorname{ch}_{\mid C}^{\text {sel }}\left(v_{x+i}\right)\right| \leq\left|\operatorname{ch}_{\mid D}^{\text {sel }}\left(v_{x+i}\right)\right| \leq F_{i}$, and $\left|\operatorname{ch}_{\mid C}^{\text {unsel }}\left(v_{x+i}\right)\right| \leq\left|\operatorname{ch}_{\mid D}^{\text {unsel }}\left(v_{x+i}\right)\right| \leq F_{i-1}$, as claimed.

We now consider the case where $\left(v_{x}, v_{x+1}\right) \in C$. Let $\mathrm{s} \in\{$ sel, unsel $\}$, let $D^{\prime}=C \cup$ $\left\{\left(v_{x+1}, v_{x+2}\right)\right\}$ and let $D^{\prime \prime}=C \cup\{d\}$. By Proposition 6.4 we have that

$$
\left|\operatorname{ch}_{\mid C}^{\mathrm{s}}\left(v_{x+i}\right)\right| \leq\left|\operatorname{ch}_{\mid D^{\prime}}^{\mathrm{s}}\left(v_{x+i}\right)\right|+\left|\operatorname{ch}_{\mid D^{\prime \prime}}^{\mathrm{s}}\left(v_{x+i}\right)\right| \text {. }
$$

By induction we thus obtain that $\left|\operatorname{ch}_{\mid C}^{\text {sel }}\left(v_{x+i}\right)\right| \leq F_{i}+F_{i-1}=F_{i+1}$, and $\left|\operatorname{ch}_{\mid C}^{\text {unsel }}\left(v_{x+i}\right)\right| \leq$ $F_{i-1}+F_{i-2}=F_{i}$, as claimed.

Recall that an active vertex $v_{i}$ with $i \geq 2$ is called pleasant if the outgoing diagonal of the previous active vertex $v_{j}$ does not point to a vertex in $\left\{v_{j+2}, \ldots, v_{i-1}\right\}$. Similarly, we call a pattern $\left(v_{f(r)}, \ldots, v_{f(r)+m}\right)$ pleasant if none of the outgoing diagonals of the vertices in $\left\{v_{f(r)+1}, \ldots, v_{f(r)+m-1}\right\}$ point to a vertex in $\left\{v_{f(r)+m+1}, \ldots, v_{f(r+1)-1}\right\}$. A pattern which is not pleasant is called unpleasant. 


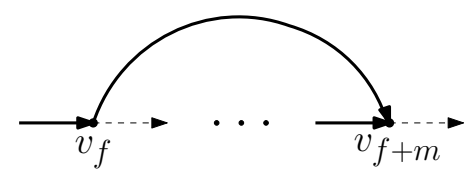

Figure 10: A choice of $\operatorname{ch}_{\mid C}^{\text {unsel }}\left(v_{f+m}\right)$.

Pleasant patterns We derive a refinement of Proposition 6.14.(c) and 6.14.(d) for pleasant patterns.

Let $1 \leq r \leq k$, let $w_{r} \geq 2$ and let $m=w_{r}$. We consider the pattern $\left(v_{f(r)}, \ldots, v_{f(r)+m}\right)$. The next proposition bounds the number of choices in $\operatorname{ch}\left(v_{f(r)+m}\right)$ containing/not containing $\left(v_{f(r)+m}, v_{f(r)+m+1}\right)$.

Proposition 6.16. Let $1 \leq r \leq k$ where $w_{r} \geq 2$, let $m=w_{r}$ and let $C, C^{\prime} \in \operatorname{ch}\left(v_{f(r)-1}\right)$ where $\left(v_{f(r)-1}, v_{f(r)}\right) \in C$ and $\left(v_{f(r)-1}, v_{f(r)}\right) \notin C^{\prime}$. We have

(i) $\left|\operatorname{ch}_{\mid C}^{\text {sel }}\left(v_{f(r)+m}\right)\right| \leq F_{m}+F_{m-2}$,

(ii) $\left|\operatorname{ch}_{\mid C}^{\text {unsel }}\left(v_{f(r)+m}\right)\right| \leq F_{m-1}$,

(iii) if $m=3$ then $\left|\operatorname{ch}_{\mid C^{\prime}}^{\text {sel }}\left(v_{f(r)+3}\right)\right|,\left|\operatorname{ch}_{\mid C^{\prime}}^{\text {unsel }}\left(v_{f(r)+3}\right)\right| \leq 1$.

Proof: Let $f:=f(r)$, let $D^{\prime}=C \cup\left\{\left(v_{f}, v_{f+1}\right)\right\}$ and let $D^{\prime \prime}=C \cup\left\{\left(v_{f}, v_{f+m}\right)\right\}$ (for an illustration of $D^{\prime}$ and $D^{\prime \prime}$ see Figure 9). We first show (i). Proposition 6.4 gives that

$$
\left|\operatorname{ch}_{\mid C}^{\text {sel }}\left(v_{f+m}\right)\right| \leq\left|\operatorname{ch}_{\mid D^{\prime}}^{\text {sel }}\left(v_{f+m}\right)\right|+\left|\operatorname{ch}_{\mid D^{\prime \prime}}^{\text {sel }}\left(v_{f+m}\right)\right| .
$$

By Observation 3.1 every choice of $\operatorname{ch}_{\mid D^{\prime}}^{\text {sel }}\left(v_{f+m}\right)$ contains $\left(v_{f+m-1}, v_{f+m}\right)$. Together with Observation 6.1 (for $j=f+m-1$ and $l=f+m$ ) and Proposition 6.15 this gives that

$$
\left|\operatorname{ch}_{\mid D^{\prime}}^{\mathrm{sel}}\left(v_{f+m}\right)\right| \leq\left|\operatorname{ch}_{\mid D^{\prime},+\left(v_{f+m-1}, v_{f+m}\right)}\left(v_{f+m}\right)\right| \leq\left|\operatorname{ch}_{\mid D^{\prime}}^{\mathrm{sel}}\left(v_{f+m-1}\right)\right| \leq F_{m} .
$$

By Observation 3.1 every choice of $\operatorname{ch}_{\mid D^{\prime \prime}}^{\text {sel }}\left(v_{f+m}\right)$ does not contain $\left(v_{f+m-1}, v_{f+m}\right)$. Similarly as above we obtain that

$$
\left|\operatorname{ch}_{\mid D^{\prime \prime}}^{\text {sel }}\left(v_{f+m}\right)\right| \leq\left|\operatorname{ch}_{\mid D^{\prime \prime},-\left(v_{f+m-1}, v_{f+m}\right)}\left(v_{f+m}\right)\right| \leq\left|\operatorname{ch}_{\mid D^{\prime \prime}}^{\text {unsel }}\left(v_{f+m-1}\right)\right| \leq F_{m-2} .
$$

Together with (29) and (30) this implies (i).

Let $E \in \operatorname{ch}_{\mid C}^{\text {unsel }}\left(v_{f+m}\right)$. By Observation 3.1 we have $\left(v_{f}, v_{f+m}\right),\left(v_{f+m-1}, v_{f+m}\right) \in E$, and therefore, $\left(v_{f}, v_{f+1}\right) \notin E$. Figure 10 shows an illustration. Hence,

$$
\left|\operatorname{ch}_{\mid C}^{\text {unsel }}\left(v_{f+m}\right)\right| \leq\left|\operatorname{ch}_{\mid D^{\prime \prime}}^{\text {unsel }}\left(v_{f+m}\right)\right| \leq\left|\operatorname{ch}_{\mid D^{\prime \prime},+\left(v_{f+m-1}, v_{f+m}\right)}\left(v_{f+m}\right)\right| \leq\left|\operatorname{ch}_{\mid D^{\prime \prime}}^{\text {sel }}\left(v_{f+m-1}\right)\right| \leq F_{m-1},
$$

which proves (ii). Finally, we show (iii). By listing all elements of $\mathrm{ch}_{\mid C^{\prime}}\left(v_{f+3}\right)$ (see Figure 11) it can be checked that $\left|\operatorname{ch}_{\mid C^{\prime}}^{\text {sel }}\left(v_{f+3}\right)\right|,\left|\operatorname{ch}_{\mid C^{\prime}}^{\text {unsel }}\left(v_{f+3}\right)\right| \leq 1$. Here we used the fact that due to Observation 3.1 no choice in $\operatorname{ch}\left(v_{f(r)+3}\right)$ contains a cycle of length smaller than $n$. 
Let $1 \leq r \leq k$ such that $w_{r} \geq 2$ and $\left(v_{f(r)}, \ldots, v_{f(r)+w_{r}}\right)$ is pleasant, let $m=w_{r}$, let $f=f(r)$ and let $f^{\prime}=f(r+1)$. We fix two choices $C, C^{\prime} \in \operatorname{ch}\left(v_{f-1}\right)$ where $\left(v_{f-1}, v_{f}\right) \in C$ and $\left(v_{f-1}, v_{f}\right) \notin C^{\prime}$. Since $\left(v_{f}, \ldots, v_{f+m}\right)$ is pleasant every incoming diagonal of a vertex in $\left\{v_{f+m+1}, \ldots, v_{f^{\prime}-1}\right\}$ has its source in $\left\{v_{1}, \ldots, v_{f-1}\right\}$. (In particular, $v_{f^{\prime}}$ is pleasant.) So Proposition 6.9 (for $i=f$ and $j=f+m$ ), Observation 6.2 and Proposition 6.16 imply that

$$
\begin{aligned}
\left|\operatorname{ch}_{\mid C}^{\text {sel }}\left(v_{f^{\prime}-1}\right)\right| & \leq \max _{\oplus \in\{+,-\}}\left(\left|\operatorname{ch}_{\mid C, \oplus\left(v_{f+m}, v_{f+m+1}\right)}\left(v_{f^{\prime}-1}\right)\right|\right) \\
& \leq \max _{\oplus \in\{+,-\}}\left(\left|\operatorname{ch}_{\mid C, \oplus\left(v_{f+m}, v_{f+m+1}\right)}\left(v_{f+m}\right)\right|\right) \leq F_{m}+F_{m-2} .
\end{aligned}
$$

Moreover, if $m=3$ we similarly get that

$$
\left|\operatorname{ch}_{\mid C^{\prime}}^{\text {sel }}\left(v_{f^{\prime}-1}\right)\right| \leq \max _{\oplus \in\{+,-\}}\left(\left|\operatorname{ch}_{\mid C^{\prime}, \oplus\left(v_{f+3}, v_{f+4}\right)}\left(v_{f+3}\right)\right|\right) \leq 1 .
$$

(31) and (32) imply the following.

Lemma 6.17. Let $1 \leq r \leq k$ such that $w_{r} \geq 2$ and $\left(v_{f(r)}, \ldots, v_{f(r)+w_{r}}\right)$ is pleasant, and let $m=w_{r}$. Moreover, let $C, C^{\prime} \in \operatorname{ch}\left(v_{f(r)-1}\right)$ where $\left(v_{f(r)-1}, v_{f(r)}\right) \in C$ and $\left(v_{f(r)-1}, v_{f(r)}\right) \notin$ $C^{\prime}$. Then

$$
\left|\operatorname{ch}_{\mid C}^{\mathrm{sel}}\left(v_{f(r+1)-1}\right)\right| \leq F_{m}+F_{m-2} .
$$

If $w_{r}=3$ then additionally, $\left|\operatorname{ch}_{\mid C^{\prime}}^{\text {sel }}\left(v_{f(r+1)-1}\right)\right| \leq 1$.

Unpleasant patterns We will need the following observation.

Observation 6.18. By induction, for every $i, j \geq 2$ we have $F_{i} \cdot F_{j} \leq 2 F_{i+j-3}$.

We now strengthen Proposition 6.14.(c) for unpleasant patterns. Recall that a pattern $\left(v_{f(r)}, \ldots, v_{f(r)+m}\right)$ is called unpleasant if some outgoing diagonal of a vertex in $\left\{v_{f(r)+1}, \ldots, v_{f(r)+m-1}\right\}$ points to a vertex in $\left\{v_{f(r)+m+1}, \ldots, v_{f(r+1)-1}\right\}$. We fix an $r$ with $1 \leq r \leq k$ such that $w_{r} \geq 2$, the vertex $v_{f(r+1)}$ is pleasant, and the pattern $\left(v_{f(r)}, \ldots, v_{f(r)+w_{r}}\right)$ is unpleasant. Let $f:=f(r)$, let $f^{\prime}:=f(r+1)$ and let $m:=w_{r}$. Moreover, let $a$ denote the largest index such that the outgoing diagonal of $v_{f+a}$ points to a vertex $v_{f+b} \in\left\{v_{f+m+1}, \ldots, v_{f^{\prime}-1}\right\}$. Figure 12 shows an illustration. We have $a \geq 1$. Since $v_{f^{\prime}}$ is pleasant we get that

$$
1 \leq a \leq m-2 .
$$

We first bound the number of choices $D \in \mathrm{ch}^{\text {sel }}\left(v_{f^{\prime}-1}\right)$ which are extensions of a given choice $C$.
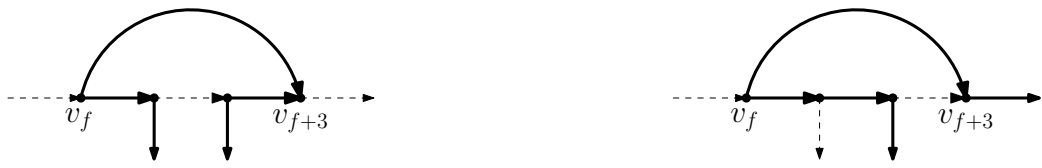

Figure 11: The two elements of $\operatorname{ch}_{\mid C^{\prime}}\left(v_{f+3}\right)$. 


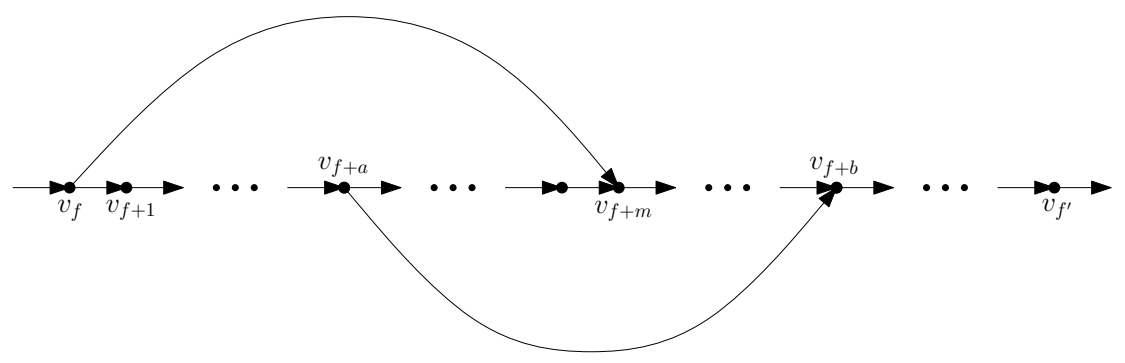

Figure 12: An unpleasant pattern.

Observation 6.19. Let $C \in \operatorname{ch}\left(v_{f+a-1}\right)$ and let $\oplus \in\{+,-\}$. Observation 3.1 gives that

$$
\left|\operatorname{ch}_{\mid C, \oplus\left(v_{f+a}, v_{f+b}\right)}\left(v_{f+a}\right)\right| \leq 1 .
$$

Proposition 6.20. Let $C \in \operatorname{ch}\left(v_{f+a}\right)$. Then $\left|\operatorname{ch}_{\mid C}^{\text {sel }}\left(v_{f^{\prime}-1}\right)\right| \leq F_{m-a}$.

Proof: Note that $l(r)=f+m-1$. By Observation 3.3, Corollary 6.10 and Proposition 6.11 we get that

$$
\left|\operatorname{ch}_{\mid C}^{\mathrm{sel}}\left(v_{f^{\prime}-1}\right)\right| \leq \sum_{D \in \mathrm{ch}_{\mid C}\left(v_{f+m-2}\right)}\left|\operatorname{ch}_{\mid D}^{\mathrm{sel}}\left(v_{f^{\prime}-1}\right)\right| \leq\left|\operatorname{ch}_{\mid C}\left(v_{f+m-2}\right)\right| \leq F_{m-a},
$$

as claimed.

We fix a choice $C \in \operatorname{ch}\left(v_{f+a-1}\right)$ where $\left(v_{f}, v_{f+m}\right) \notin C$. Our goal is to show that Proposition 6.20 also holds for this $C$. Let $D \in \operatorname{ch}_{\mid C}^{\text {sel }}\left(v_{f^{\prime}-1}\right)$. Similarly to the proof of Proposition 6.9, we aim to show that many properties of $D$ are uniquely determined by C.

Observation 6.21. Let $d$ be an incoming diagonal of a vertex in $\left\{v_{f+m}, \ldots, v_{f+b-1}\right\} \cup$ $\left\{v_{f+b+1}, \ldots, v_{f^{\prime}-1}\right\}$. By our choice of a the diagonal d has its source in $\left\{v_{1}, \ldots, v_{f+a-1}\right\}$. In particular, it holds that $d \in D$ if and only if $d \in C$.

Let

$$
j:=\max _{i<b}\left(i \text { : the incoming diagonal of } v_{f+i} \text { does not belong to } C\right) .
$$

Note that by assumption the diagonal $\left(v_{f}, v_{f+m}\right)$ does not belong to $C$, thus $j \geq m$. By Observation 6.21 and (34) we have that (i) the incoming diagonal of $v_{f+j}$ does not belong to $D$, and (ii) for every $j+1 \leq i \leq b-1$ the incoming diagonal of $v_{f+i}$ belongs to $D$. By Observation 3.1 we obtain that $\left(v_{f+j}, v_{f+j+1}\right) \in D$. Together with Observation 6.3 (for $i_{\text {start }}=f+j$ and $\left.i_{\text {end }}=f+b\right)$ we get that

$$
\left(v_{f+b-1}, v_{f+b}\right) \in D \Leftrightarrow b-j \equiv 1 \quad(\bmod 2) .
$$

By Proposition 6.9 (for $i=f+a$ and $j=f+b$ ) there is a value $g(C) \in\{0,1\}$ (where $g$ is a function of $C$ only) such that

$$
\left(v_{f+b}, v_{f+b+1}\right) \in D \Leftrightarrow g(C)=1 .
$$


By Observation 3.1 we have that

$$
\left(v_{f+a}, v_{f+b}\right) \in D \Leftrightarrow\left(v_{f+b-1}, v_{f+b}\right) \notin D \text { or }\left(v_{f+b}, v_{f+b+1}\right) \notin D .
$$

Let $g^{\prime}(C)=\left\{\begin{array}{l}1, \text { if } g(C)=0 \text { or } b-j \equiv 0(\bmod 2) \\ 0, \text { otherwise }\end{array}\right.$

Note that $g^{\prime}$ is a function of $C$ only. So by (35) - (37) every $D \in \operatorname{ch}_{\mid C}^{\text {sel }}\left(v_{f^{\prime}-1}\right)$ has the property that

$$
\left(v_{f+a}, v_{f+b}\right) \in D \Leftrightarrow g^{\prime}(C)=1
$$

Hence,

$$
\left|\operatorname{ch}_{\mid C}^{\text {sel }}\left(v_{f^{\prime}-1}\right)\right| \leq \max _{\oplus \in\{+,-\}}\left(\left|\operatorname{ch}_{\mid C, \oplus\left(v_{f+a}, v_{f+b}\right)}^{\text {sel }}\left(v_{f^{\prime}-1}\right)\right|\right) .
$$

Let $\oplus \in\{+,-\}$. According to Observation 6.19 there is at most one extension $D \in$ $\operatorname{ch}_{\mid C, \oplus\left(v_{f+a}, v_{f+b}\right)}\left(v_{f+a}\right)$. Together with Proposition 6.20 this implies that

$$
\left|\operatorname{ch}_{\mid C, \oplus\left(v_{f+a}, v_{f+b}\right)}^{\text {sel }}\left(v_{f^{\prime}-1}\right)\right| \leq\left|\operatorname{ch}_{\mid D}^{\text {sel }}\left(v_{f^{\prime}-1}\right)\right| \leq F_{m-a} .
$$

By (38) this shows the next proposition.

Proposition 6.22. Let $C \in \operatorname{ch}\left(v_{f+a-1}\right)$ where $\left(v_{f}, v_{f+m}\right) \notin C$. Then $\left|\operatorname{ch}_{\mid C}^{\mathrm{sel}}\left(v_{f^{\prime}-1}\right)\right| \leq F_{m-a}$. Proposition 6.22 allows us to derive a lemma similar to Lemma 6.17. Let $C \in \operatorname{ch}\left(v_{f-1}\right)$ where $\left(v_{f-1}, v_{f}\right) \in C$. Proposition 6.4 gives that

$$
\left|\operatorname{ch}_{\mid C}^{\text {sel }}\left(v_{f^{\prime}-1}\right)\right| \leq\left|\operatorname{ch}_{\mid D^{\prime}}^{\text {sel }}\left(v_{f^{\prime}-1}\right)\right|+\left|\operatorname{ch}_{\mid D^{\prime \prime}}^{\text {sel }}\left(v_{f^{\prime}-1}\right)\right|,
$$

where $D^{\prime}=C \cup\left\{\left(v_{f}, v_{f+1}\right)\right\}$ and $D^{\prime \prime}=C \cup\left\{\left(v_{f}, v_{f+m}\right)\right\}$. By Observation 3.3, Corollary 6.10 (using that $l(r)=f+m-1$ ) and Proposition 6.11 we get that

$$
\left|\operatorname{ch}_{\mid D^{\prime \prime}}^{\mathrm{sel}}\left(v_{f^{\prime}-1}\right)\right| \leq \sum_{E \in \mathrm{ch}_{\mid D^{\prime \prime}}\left(v_{f+m-2}\right)}\left|\operatorname{ch}_{\mid E}^{\mathrm{sel}}\left(v_{f^{\prime}-1}\right)\right| \leq\left|\operatorname{ch}_{\mid D^{\prime \prime}}\left(v_{f+m-2}\right)\right| \leq F_{m-1} .
$$

By Observation 3.3, Proposition 6.22 and Proposition 6.11 we obtain that

$$
\begin{aligned}
\left|\operatorname{ch}_{\mid D^{\prime}}^{\mathrm{sel}}\left(v_{f^{\prime}-1}\right)\right| & \leq \sum_{E \in \mathrm{ch}_{\mid D^{\prime}}\left(v_{f+a-1}\right)}\left|\operatorname{ch}_{\mid E}^{\mathrm{sel}}\left(v_{f^{\prime}-1}\right)\right| \\
& \leq \sum_{E \in \mathrm{ch}_{\mid D^{\prime}}\left(v_{f+a-1}\right)} F_{m-a} \\
& \leq\left|\operatorname{ch}_{\mid D^{\prime}}\left(v_{f+a-1}\right)\right| \cdot F_{m-a} \leq F_{a+1} \cdot F_{m-a} .
\end{aligned}
$$

(39) - (41) together with (33) and Observation 6.18 give that

$$
\left|\operatorname{ch}_{\mid C}^{\text {sel }}\left(v_{f^{\prime}-1}\right)\right| \leq F_{m-1}+F_{a+1} \cdot F_{m-a} \leq F_{m-1}+2 F_{m-2}=F_{m}+F_{m-2} .
$$

We observe that, if $m=3$ then by (33) we have that $a=1$, and therefore $\left|\operatorname{ch}_{\mid C}^{\text {sel }}\left(v_{f^{\prime}-1}\right)\right| \leq 2$. Thus (42) directly implies the following. 
Lemma 6.23. Let $1 \leq r \leq k$ such that $w_{r} \geq 2$, the pattern $\left(v_{f(r)}, \ldots, v_{f(r)+w_{r}}\right)$ is unpleasant, and the vertex $v_{f(r+1)}$ is pleasant. Moreover, let $m=w_{r}$ and let $C \in \operatorname{ch}\left(v_{f(r)-1}\right)$ where $\left(v_{f(r)-1}, v_{f(r)}\right) \in C$. Then

$$
\left|\operatorname{ch}_{\mid C}^{\mathrm{sel}}\left(v_{f(r+1)-1}\right)\right| \leq F_{m}+F_{m-2} .
$$

If $w_{r}=3$ then additionally, $\left|\operatorname{ch}_{\mid C}^{\text {sel }}\left(v_{f(r+1)-1}\right)\right| \leq 2$.

Putting Lemma 6.17 and Lemma 6.23 together The next corollary is the union of Lemma 6.17 and Lemma 6.23.

Corollary 6.24. Let $1 \leq r \leq k$ such that $w_{r} \geq 2$ and $v_{f(r+1)}$ is pleasant. Then for every $C \in \operatorname{ch}\left(v_{f(r)-1}\right)$ where $\left(v_{f(r)-1}, v_{f(r)}\right) \in C$,

$$
\left|\operatorname{ch}_{\mid C}^{\text {sel }}\left(v_{f(r+1)-1}\right)\right| \leq F_{w_{r}}+F_{w_{r}-2} .
$$

If $w_{r}=3$ then, additionally, either

(i) $\left|\operatorname{ch}_{\mid C^{\prime}}^{\text {sel }}\left(v_{f(r+1)-1}\right)\right| \leq 1$, for every $C^{\prime} \in \operatorname{ch}\left(v_{f(r)-1}\right)$ where $\left(v_{f(r)-1}, v_{f(r)}\right) \notin C^{\prime}$, or

(ii) $\left|\operatorname{ch}_{\mid C}^{\text {sel }}\left(v_{f(r+1)-1}\right)\right| \leq 2$, for every $C \in \operatorname{ch}\left(v_{f(r)-1}\right)$ where $\left(v_{f(r)-1}, v_{f(r)}\right) \in C$.

\subsection{Derivation of Key Lemma 1}

Observation 6.25. If $w_{r}=1$ then $f(r)=l(r)$.

We fix an $r$ with $1 \leq r \leq k$. By Observation 3.3 (for $i=f(r)-1$ and $j=f(r+1)-1$ ) we can obtain $\left|\operatorname{ch}\left(v_{f(r+1)-1}\right)\right|$ by summing up $\left|\operatorname{ch}_{\mid C}\left(v_{f(r+1)-1}\right)\right|$ over each choice $C \in \operatorname{ch}\left(v_{f(r)-1}\right)$.

Suppose first that $w_{r}=1$. By Proposition 6.6 and Observation 6.25, every choice $C \in \operatorname{ch}\left(v_{f(r)-1}\right)$ where $\left(v_{f(r)-1}, v_{f(r)}\right) \in C$ contributes at most two to $\left|\operatorname{ch}\left(v_{f(r+1)-1}\right)\right|$, and every choice $C^{\prime} \in \operatorname{ch}\left(v_{f(r)-1}\right)$ where $\left(v_{f(r)-1}, v_{f(r)}\right) \notin C^{\prime}$ contributes at most one to $\left|\operatorname{ch}\left(v_{f(r+1)-1}\right)\right|$. Due to the definition of $A_{r}$ and $B_{r}$ this directly implies (6). If $v_{f(r+1)}$ is pleasant then by Corollary 6.10 every choice $C \in \operatorname{ch}\left(v_{f(r)-1}\right)$ contributes at most one to $\left|\mathrm{ch}^{\text {sel }}\left(v_{f(r+1)-1}\right)\right|$, which proves $(7)$.

Suppose now that $w_{r} \geq 2$. By a similar argument as before, Proposition 6.14.(a) and 6.14.(b) directly imply (8). Accordingly, Proposition 6.14.(c) and 6.14.(d) imply that

$$
A_{r} \leq F_{w_{r}+1} \cdot A_{r-1}+F_{w_{r}} \cdot B_{r-1},
$$

if $v_{f(r+1)}$ is pleasant. We remark that by proceeding along similar lines as in the proof of Key Lemma 2 we can show that Key Lemma 2 remains true if we replace (i) "(6) - (9) and (c)" with "(6) - (8) and (44)", and (ii) "1.628" with "1.64". This already implies a weaker version of Theorem 1.1 where "1.628" is replaced with "1.64".

By Corollary 6.24 we can improve (44): (43) and Proposition 6.14.(d) imply (9), and together with the second part of Corollary 6.24 they also give (c). This concludes the proof of Key Lemma 1.

Acknowledgment: We would like to thank Tibor Szabó and Emo Welzl for suggesting such a rich and beautiful topic and also for the numerous helpful discussions. 


\section{References}

[1] A. Björklund, T. Husfeldt, P. Kasaki and M. Koivisto. The Travelling Salesman Problem in Bounded Degree Graphs. Proc. 35th International Colloquium on Automata, Languages, and Programming (ICALP), Part I. Lecture Notes in Computer Science 5125, (2008), 198-209.

[2] A. Björklund. Determinant sums for undirected Hamiltonicity. Proc. 51st Annual IEEE Symposium on Foundations of Computer Science (FOCS), (2010), 173-182.

[3] D. Eppstein. The Traveling Salesman Problem for Cubic Graphs. J. Graph Algorithms and Applications 11(1), (2007), 61-68.

[4] M.R. Garey and D.S. Johnson. Computers and Intractability: A Guide to the Theory of NP-Completeness. W.H. Freeman, 1979.

[5] H. Gebauer. Finding and Enumerating Hamilton Cycles in 4-Regular Graphs. Theoretical Computer Science, to appear.

[6] M. Held and R.M. Karp. A dynamic programming approach to sequencing problems. SIAM Journal on Applied Mathematics 10, (1962), 196-210.

[7] K. Iwama and T. Nakashima. An Improved Exact Algorithm for Cubic Graph TSP. Proc. 13th Annual International Computing and Combinatorics Conference (COCOON), 4598 (2007), 108-117.

[8] M.Sharir, E.Welzl. On the number of crossing-free matchings, (cycles, and partitions). Proc. 17th Annual ACM-SIAM Symposium on Discrete Algorithms (SODA), (2006), 860-869.

[9] G.J. Woeginger. Exact Algorithms for NP-Hard Problems: A Survey. Combinatorial optimization - Eureka! you shrink! Lecture Notes in Computer Science 2570, (2003), $185-207$. 